\title{
Ist ärztliche Komplementärmedizin wirtschaftlich?
}

\section{Hans-Peter Studer ${ }^{a}$ \\ André Busato ${ }^{b}$}

Im Namen der Union schweizerischer komplementärmedizinischer Ärzteorganisationen

a Dr. oec., selbständiger Gesundheitsökonom

b Prof. Dr. Msc., Institut für Evaluative Forschung in der Orthopädie der Universität Bern

Eine ausführliche Fassung dieses Artikels inklusive Literaturangaben findet sich im Internet unter www.saez.ch $\rightarrow$ Aktuelle Nummer oder $\rightarrow$ Archiv $\rightarrow 2010 \rightarrow 18$

Interessenbindungen: Die vorliegenden Daten wurden im Rahmen des Projekts Programm Evaluation Komplementärmedizin erhoben, das vom Bundesamt für Gesundheit (BAG) finanziert wurde. Beide Autoren waren seinerzeit als Projektmitarbeiter mit der Prüfung der Wirtschaftlichkeit der ärztlichen Komplementärmedizin betraut. Das BAG nahm keinerlei Einfluss auf die Publikation dieses Artikels. Die Autoren unterliegen keinen Interessenbindungen.

Korrespondenz:

Dr. oec. Hans-Peter Studer Rickstrasse 31

CH-9037 Speicherschwendi

Tel. 0713443837

Fax 0713443840

hpstuder@swissonline.ch

\section{Einleitung}

Während sechs Jahren waren fünf Methoden der ärztlichen Komplementärmedizin provisorisch im Grundleistungskatalog der obligatorischen Krankenpflegeversicherung der Schweiz enthalten. Im Juni 2005 entschied der damalige Bundesrat und Gesundheitsminister Pascal Couchepin, sie wieder zu entfernen, mit der Begründung, dass die Wirksamkeit, Zweckmässigkeit und Wirtschaftlichkeit dieser Methoden nicht nachgewiesen werden konnten. Das führte seinerzeit zu erheblichen Irritationen, unter anderem auch beim internationalen Review Board, welches das PEK-Forschungsprogramm wissenschaftlich begleitet hatte.

Zwischenzeitlich hat die schweizerische Bevölkerung dem parlamentarischen Gegenvorschlag zur Volksinitiative «Ja zur Komplementärmedizin» zugestimmt, der u. a. mit der Forderung verbunden war, die ärztliche Komplementärmedizin wieder in die Grundversicherung aufzunehmen. Um die Berechtigung dieses Anliegens beurteilen zu können, ist es naheliegend, das umfangreiche Datenmaterial des PEK-Forschungsprogramms zu Rate zu ziehen. Ziel dieses Artikels ist, die PEK-Daten zur wichtigen Frage der Wirtschaftlichkeit der Komplementärmedizin in einem Gesamtkontext darzustellen und zu würdigen.

\section{Miteinbezogene Studien}

Für das PEK-Forschungsprojekt stellte santésuisse, der Dachverband der Schweizer Krankenversicherer, Kostendaten für die Jahre 2002 und 2003 zur Verfügung, welche sich auf die an der PEK-Praxisstudie I beteiligten Ärzte bezogen. Sie beinhalteten einerseits die Kosten, die ein Arzt pro Jahr in seiner Praxis generierte (konsultationsbezogene Kosten). Diese setzen sich zusammen aus den Konsultationskosten und den Kosten für direkt in der Praxis abgegebene Medikamente. Bei den Kosten, die andererseits durch den betreffenden Arzt ausserhalb seiner Praxis veranlasst wurden (veranlasste Kosten), liessen sich aufgrund der santésuisse-Datenbank nur drei Kategorien zuverlässig dem einzelnen Arzt zuordnen: Kosten für Medikamente, die auf ärztliches Rezept in einer Apotheke bezogen wurden, Kosten für externe Laboruntersuchungen und Kosten für verordnete Physiotherapien.

Diese Kostendaten ermöglichten einen Vergleich zwischen den 174 rein schulmedizinisch respektive konventionell tätigen Ärzten (COM-Ärzte), die an der Praxisstudie I teilgenommen hatten, mit den 151 Ärzten, die in ihrer Praxis auch Komplementärmedizin einsetzten, aber nicht über einen entsprechenden

\section{La médecine complémentaire dispensée par des médecins est-elle économique?}

Les données sur les coûts, les médecins et les patients récoltées dans le cadre du «Programme d'évaluation des médecines complémentaires» (PEK) a permis de mettre en évidence les points suivants: la durée des consultations auprès de médecins qui, outre leur cursus en médecine classique ont suivi une formation en médecine complémentaire et acquis une attestation de formation dans ce domaine, est certes plus longue mais les coûts par patient et par an imputés à l'assurance obligatoire des soins ne sont pas plus élevés. Les coûts calculés par médecin et par an sont même inférieurs de $29 \%$. Les médecins au bénéfice d'une attestation FMH de formation complémentaire proposent des soins de base centrés sur les patients ce qui entraîne une plus grande satisfaction de ces derniers.

FMH-anerkannten Fähigkeitsausweis verfügten (nichtzertifizierte CAM-Ärzte) und den 257 Ärzten, die zusätzlich zur Schulmedizin auch Komplementärmedizin einsetzten und hierfür über einen entsprechenden Fähigkeitsausweis verfügten (zertifizierte CAM-Ärzte).

Für die Beurteilung der Wirtschaftlichkeit massgebend ist neben den Kosten auch der Nutzen einer Therapiemethode. Die vorliegenden Daten erlauben zwar keine direkten Kosten-Nutzen-Analysen, aber es lassen sich in Bezug auf eine Kosten-WirksamkeitsAnalyse Aussagen machen, aufgrund derer sich die Wirtschaftlichkeit der ärztlichen Komplementärmedizin abschätzen lässt.

Grundlage hierfür bildet zum einen die Praxisstudie II, bei der über einen Zeitraum von einem Jahr bei 318 Ärzten (71 COM-Ärzte, 77 nicht-zertifizierte CAMÄrzte, 170 zertifizierte CAM-Ärzte), die an der Praxisstudie I teilgenommen hatten, an vier saisonalen Stichtagen systematisch insgesamt 11615 Behandlungen von Patienten dokumentiert wurden, die älter als 16 Jahre waren. Weiter standen Daten von 6133 Patienten zur Verfügung, die einen Monat nach der Behandlung einen Fragebogen beantwortet hatten. Aus diesen Befragungen ergeben sich unter anderem Hinweise auf den Behandlungserfolg und die Zufrieden- 
heit mit der Behandlung, aber auch Angaben zu den Nebenwirkungen sowie zu den mit der Erkrankung verbundenen Arbeitsausfalltagen.

\section{Ergebnisse}

\section{Kosten pro Arzt}

Der Vergleich der empirischen Daten ergab pro Arzt grosse Unterschiede bei den jährlich zu Lasten der obligatorischen Krankenpflegeversicherung generierten Kosten. Wie Tabelle 1 zeigt, sind die beobachteten Kosten bei den zertifizierten CAM-Ärzten durchweg am tiefsten und bei den rein schulmedizinisch tätigen COM-Ärzten durchweg am höchsten. Dazwischen liegen die Kosten bei denjenigen Ärzten, die Komplementärmedizin ohne entsprechenden Fähigkeitsausweis zusätzlich zur Schulmedizin einsetzten (nichtzertifizierte CAM-Ärzte).

Besonders gross sind die Unterschiede bei den veranlassten Kosten, etwas weniger ausgeprägt bei den konsultationsbezogenen Kosten (inkl. direkt abgegebene Medikamente). Der Mittelwert der gesamten Medikamentenkosten, die sich aus den Kosten für direkt in der Praxis abgegebene Medikamente und denjenigen für verschriebene Medikamente zusammensetzen, beläuft sich bei den zertifizierten CAM-Ärzten lediglich auf ein Drittel desjenigen der COM-Ärzte. Was den Mittelwert der Gesamtkosten anbelangt, so ist er bei zertifizierten CAM-Ärzten genau halb so hoch wie bei den COM-Ärzten.

Allerdings gilt es zu beachten, dass die Zusammensetzung der Patientenkollektive in den jeweiligen Arztpraxen verschieden war, und dass sich die Praxen auch von der Struktur her unterschieden. Entsprechend wurden die empirischen Kosten mit einem nicht-parametrischen Regressionsmodell für neun Variablen statistisch korrigiert: Ort der Praxis nach Kanton, Urbanisationsgrad des Praxisorts, Art der Praxis (Einzeloder Gruppenpraxis), Geschlecht des Arztes, Anzahl der Jahre, in denen der Arzt nach dem Staatsexamen berufstätig war, Anteil weiblicher Patienten, Durch- schnittsalter der Patienten, Anteil Hausbesuche, Anteil unfallbezogener Konsultationen.

Daraus resultierten pro Arztpraxis und Jahr modellbasierte Kosten gemäss Tabelle 1. Auch hier unterscheiden sich die Kosten zertifizierter CAM-Ärzte signifikant von denjenigen der nicht-zertifizierten CAM-Ärzte und der COM-Ärzte. Bei allen Kostenkategorien liegen sie unter denjenigen der beiden anderen Gruppen. Die modellbasierten Gesamtkosten der zertifizierten CAMÄrzte liegen um 29\% tiefer als diejenigen der COMÄrzte, die separat ausgewiesenen Medikamentenkosten um $38,5 \%$ tiefer.

\section{Kosten pro Patient}

Die Kosten pro Patient errechnen sich aus den Kosten pro Arzt dividiert durch die Anzahl der in den Jahren 2002 und 2003 behandelten Patienten. Diese war in den drei Gruppen sehr unterschiedlich: ein zertifizierter CAM-Arzt behandelte im Durchschnitt pro Jahr deutlich weniger - nämlich 648 - Patienten als ein nicht-zertifizierter CAM-Arzt (946 Patienten) oder ein COM-Arzt (988 Patienten). Der Hauptgrund dafür war, dass die zertifizierten CAM-Ärzte pro Konsultation erheblich mehr Zeit aufwendeten (29,1 Min., nicht-zertifizierte CAM-Ärzte: 21,1 Min., COM-Ärzte: 17,1 Min.). Zudem lag bei ihnen die Zahl der Konsultationen pro Patient und Jahr etwas höher.

Wie Tabelle 2 zeigt, sind folglich die empirischen Konsultationskosten pro Patient und Jahr bei zertifizierten CAM-Ärzten höher als bei nicht-zertifizierten CAM-Ärzten und bei COM-Ärzten. Im Gegensatz dazu sind jedoch die veranlassten Kosten und insbesondere die Medikamentenkosten im Vergleich deutlich tiefer, was die höheren Konsultationskosten mehr als kompensiert. Entsprechend liegen die patientenbezogenen Gesamtkosten pro Jahr bei den zertifizierten CAMÄrzten am tiefsten und bei den COM-Ärzten am höchsten.

Um den Unterschieden in der Zusammensetzung der Patientenkollektive und in der Struktur der Praxis Rechnung zu tragen, wurden auch die empirischen

\section{Tabelle 1}

Jährliche Kosten (in CHF) zu Lasten der obligatorischen Krankenpflegeversicherung von zertifizierten und nicht-zertifizierten komplementärmedizinisch tätigen Ärzten (CAM-Ärzten) sowie von rein schulmedizinisch tätigen Ärzten (COM-Ärzten), 2002/2003, ohne Kostenselbstbehalte der Patienten.

Art der Kosten (in CHF)

Art der Kosten (in CHF)

\section{Konsultationsbezogene Kosten}

Gesamtkosten

davon: Medikamentenkosten

Zertifizierte CAM-Ärzte
\begin{tabular}{ll} 
Empirische & Modellbasierte \\
Daten & Daten \\
\hline Mittelwert & LS-mean $^{1} \quad$ SE$^{2}$
\end{tabular}

298400

118800

417200

386100

142700

$531600^{a}$

$229900^{a}$

$+37400$

Nicht-zertifizierte CAM-Ärzte

Empirische Modellbasierte

Daten Daten

Mittelwert LS-mean SE

$154900^{\mathrm{a}} \pm 28300$

Mittelwert

435000

4 S-mean

Veranlasste Kosten

a,b Unterschiedliche Buchstaben weisen auf signifikante Unterschiede zwischen den Gruppen hin $(p<0,05)$

1 Modellbasierter Mittelwert, errechnet mittels nicht-parametrischer Regression (Least Square Mean).

2 Standardfehler

$3 r^{2}=$ Anteil der durch das statistische Modell erklärten Varianz der Zielvariablen.

\begin{tabular}{|c|c|c|c|}
\hline COM-Ärzte & & & $r^{23}$ \\
\hline $\begin{array}{l}\text { Empirische } \\
\text { Daten }\end{array}$ & $\begin{array}{l}\text { Modellba } \\
\text { Daten }\end{array}$ & & \\
\hline Mittelwert & LS-mean & SE & \\
\hline 457900 & $436800^{b}$ & \pm 38400 & $39,5 \%$ \\
\hline 376500 & $301600^{b}$ & \pm 29000 & $47,7 \%$ \\
\hline 834400 & $748700^{b}$ & \pm 50100 & $42,8 \%$ \\
\hline 441400 & $373900^{b}$ & \pm 36000 & $40,5 \%$ \\
\hline
\end{tabular}

\begin{tabular}{llllll}
$428400^{\mathrm{b}}$ & \pm 37900 & 457900 & $436800^{\mathrm{b}}$ & \pm 38400 & $39,5 \%$ \\
$228400^{\mathrm{b}}$ & \pm 28900 & 376500 & $301600^{\mathrm{b}}$ & \pm 29000 & $47,7 \%$ \\
$664200^{\mathrm{b}}$ & \pm 49600 & 834400 & $748700^{\mathrm{b}}$ & \pm 50100 & $42,8 \%$ \\
$309000^{\mathrm{b}}$ & \pm 36300 & 441400 & $373900^{\mathrm{b}}$ & \pm 36000 & $40,5 \%$ \\
\hline
\end{tabular}




\section{Tabelle 2}

Kosten pro Patient (in CHF) zu Lasten der obligatorischen Krankenpflegeversicherung bei zertifizierten und nicht-zertifizierten komplementärmedizinisch tätigen Ärzten (CAM-Ärzten) sowie bei rein schulmedizinisch tätigen Ärzten (COM-Ärzten), 2002/2003, ohne Kostenselbstbehalte der Patienten.

\begin{tabular}{|c|c|c|c|c|c|c|c|c|c|c|}
\hline \multirow[t]{3}{*}{ Art der Kosten (in CHF) } & \multicolumn{3}{|c|}{ Zertifizierte CAM-Ärzte } & \multicolumn{3}{|c|}{ Nicht-zertifizierte CAM-Ärzte } & \multicolumn{3}{|l|}{ COM-Ärzte } & \multirow[t]{3}{*}{$r^{23}$} \\
\hline & \multirow{2}{*}{$\begin{array}{l}\text { Empirische } \\
\text { Daten } \\
\text { Mittelwert }\end{array}$} & \multicolumn{2}{|c|}{$\begin{array}{l}\text { Modellbasierte } \\
\text { Daten }\end{array}$} & \multirow{2}{*}{$\begin{array}{l}\text { Empirische } \\
\text { Daten } \\
\text { Mittelwert }\end{array}$} & \multicolumn{2}{|c|}{$\begin{array}{l}\text { Modellbasierte } \\
\text { Daten }\end{array}$} & \multirow{2}{*}{$\begin{array}{l}\text { Empirische } \\
\text { Daten } \\
\text { Mittelwert }\end{array}$} & \multicolumn{2}{|c|}{$\begin{array}{l}\text { Modellbasierte } \\
\text { Daten }\end{array}$} & \\
\hline & & LS-mean ${ }^{1}$ & $\mathrm{SE}^{2}$ & & LS-mean & SE & & LS-mean & SE & \\
\hline Konsultationsbezogene Kosten & 517 & $452^{\mathrm{a}}$ & \pm 44 & 474 & $437 \mathrm{~b}$ & \pm 44 & 473 & $403^{b}$ & \pm 44 & $39,5 \%$ \\
\hline Veranlasste Kosten & 190 & $191^{\mathrm{a}}$ & \pm 57 & 312 & $245^{b}$ & \pm 58 & 458 & $280^{b}$ & \pm 58 & $54,2 \%$ \\
\hline Gesamtkosten & 708 & $661^{\mathrm{a}}$ & \pm 57 & 786 & $730^{\mathrm{b}}$ & \pm 57 & 931 & $709^{b}$ & \pm 58 & $55,5 \%$ \\
\hline davon: Medikamentenkosten & 197 & $254^{a}$ & \pm 48 & 377 & $330^{\mathrm{b}}$ & \pm 48 & 481 & $333^{b}$ & +48 & $65,5 \%$ \\
\hline
\end{tabular}

$a$, b Unterschiedliche Buchstaben weisen auf signifikante Unterschiede zwischen den Gruppen hin $(p<0,05)$.

1 Modellbasierter Mittelwert, errechnet mittels nicht-parametrischer Regression (Least Square Mean).

2 Standardfehler

$3 r^{2}=$ Anteil der durch das statistische Modell erklärten Varianz der Zielvariablen.

Kosten pro Patient und Jahr mit dem gleichen nichtparametrischen Regressionsmodell statistisch adjustiert. Wie Tabelle 2 zeigt, ergaben sich in der Folge signifikant höhere konsultationsbezogene Kosten pro Patient der zertifizierten CAM-Ärzte im Vergleich zu den nicht-zertifizierten CAM-Ärzten und den COM-Ärzten. Umgekehrt waren die Kosten bei den zertifizierten CAM-Ärzten signifikant tiefer als diejenigen der nichtzertifizierten CAM-Ärzte und der COM-Ärzte.

Diese beiden gegenläufigen Tendenzen heben sich bei den Gesamtkosten auf. Sie sind zwar bei den zertifizierten CAM-Ärzten am tiefsten, gefolgt von denjenigen der COM-Ärzte und jenen der nicht-zertifizierten CAM-Ärzte. Die Unterschiede sind jedoch statistisch nicht signifikant, das heisst die patientenbezogenen Kosten der drei Ärztegruppen sind bei der gegebenen Stichprobengrösse hinsichtlich der miteinbezogenen Kostenkategorien gleich. Signifikante Kostenunterschiede ergeben sich demgegenüber bei den separat ausgewiesenen Medikamentenkosten pro Patient.

\section{Unterschiedlicher Gesundheitszustand der Patienten}

In die statistische Korrektur der Kostendaten bewusst nicht miteinbezogen wurde der unterschiedliche Ge-

\section{Tabelle 3}

Schweregrad der Erkrankung, erwachsene Patienten, 2002/2003.

\begin{tabular}{|c|c|c|c|}
\hline & $\begin{array}{l}\text { Zertifizierte } \\
\text { CAM-Ärzte }\end{array}$ & $\begin{array}{l}\text { Nicht-zertifizierte } \\
\text { CAM-Ärzte }\end{array}$ & COM-Ärzte \\
\hline $\begin{array}{l}\text { Anteil schwerwiegender Gesundheits- } \\
\text { probleme (Hauptsymptom), } \\
\text { Selbsteinschätzung Patient }\end{array}$ & $26 \%$ & $22 \%$ & $20 \%$ \\
\hline $\begin{array}{l}\text { Anteil schwerwiegender Gesundheits- } \\
\text { probleme (Hauptsymptom), } \\
\text { Einschätzung Arzt }\end{array}$ & $18 \%$ & $13 \%$ & $10 \%$ \\
\hline $\begin{array}{l}\text { Anteil lebensbedrohlicher Erkrankungen } \\
\text { (Charlson-Komorbiditäts-Index }>0 \text { ) }\end{array}$ & $6 \%$ & $9 \%$ & $11 \%$ \\
\hline $\begin{array}{l}\text { Anteil chronischer Erkrankungen } \\
\text { (> } 3 \text { Monate) }\end{array}$ & $62 \%$ & $47 \%$ & $46 \%$ \\
\hline
\end{tabular}

sundheitszustand der Patienten. Einerseits wurde er als intrinsische Komponente des Angebots und der Nachfrage innerhalb einer spezifischen Behandlungsphilosophie erachtet. Andererseits ist der Gesundheitszustand der Patienten auf Niveau Praxis schwierig zu bestimmen. Er ergibt sich unter anderem aus der Selbsteinschätzung der Patienten und der Einschätzung der behandelnden Ärzte, aus der Anzahl und dem Schweregrad von Komorbiditäten sowie aus dem Anteil chronischer Erkrankungen.

Wie Tabelle 3 zeigt, ist der Schweregrad des Hauptsymptoms sowohl in der eigenen Einschätzung der Patienten als auch in derjenigen der behandelnden Ärzte bei den Patienten der zertifizierten CAM-Ärzte am grössten und bei den COM-Ärzten deutlich geringer. Dazwischen liegen die Werte für die Patienten der nicht-zertifizierten CAM-Ärzte. Umgekehrt ist der mit dem Charlson-Komorbiditäts-Index gemessene Anteil der lebensbedrohlichen Erkrankungen bei Patienten von COM-Ärzten am grössten und bei den zertifizierten CAM-Ärzten am geringsten. Ausgeprägt sind die Unterschiede auch hinsichtlich des Anteils von Patienten mit chronischen Erkrankungen. Er liegt bei den zertifizierten CAM-Ärzten deutlich höher als derjenige bei den nicht-zertifizierten CAM-Ärzten und den COM-Ärzten.

Je schwerer die Erkrankung eines Patienten ist, desto höher sind in der Regel die damit verbundenen Gesundheitskosten. Was den Schweregrad anbelangt, so ergibt sich ein unterschiedliches Bild, je nachdem, ob man die Selbsteinschätzung des Patienten und die Einschätzung des Arztes oder die Lebensbedrohlichkeit einer Erkrankung zugrunde legt. Es lässt sich also aufgrund der vorliegenden Daten und Analysen keine klare Aussage darüber machen, wie sich die mit dem Schweregrad einer Erkrankung verbundenen Kosten in den drei untersuchten Gruppen verhalten. Was jedoch den Anteil chronisch Kranker anbelangt, so unterscheiden sich die statistisch adjustierten Kosten von zertifizierten CAM-Ärzten deutlich von denjenigen von COM-Ärzten. Je grösser der Anteil chronisch 
Kranker bei COM-Ärzten ist, desto höher sind ihre Kosten. Bei den zertifizierten CAM-Ärzten ist dies nicht der Fall (siehe Abb. 1).

\section{Nutzenbezogene Ergebnisse}

Wie Tabelle 4 zeigt, ist der Anteil der Patienten, die einen Monat nach der dokumentierten Behandlung angeben, ihre Beschwerden seien nicht mehr vorhanden oder wesentlich schwächer, in allen drei untersuchten Gruppen in etwa gleich hoch. Das Gleiche gilt für die vollkommene Erfüllung der Erwartungen an die Behandlung. Die Patienten von zertifizierten und von nicht-zertifizierten CAM-Ärzten sind jedoch in höherem Masse sehr zufrieden mit dem bisherigen Verlauf der Behandlung als die Patienten von COMÄrzten. Das entspricht dem Bild, das die Beurteilung der Qualität der Beziehung zwischen Arzt und Patient mittels Europep-Fragebogen ergab. Es fiel vor allem für die Bereiche «Kommunikation und Arzt-Patienten-

\section{Abbildung 1}

Statistisch adjustierte Kosten in Abhängigkeit vom Anteil chronischer Patienten, Vergleich COM-Ärzte und zertifizierte CAM-Ärzte, logarithmische Skala, 2002/2003.

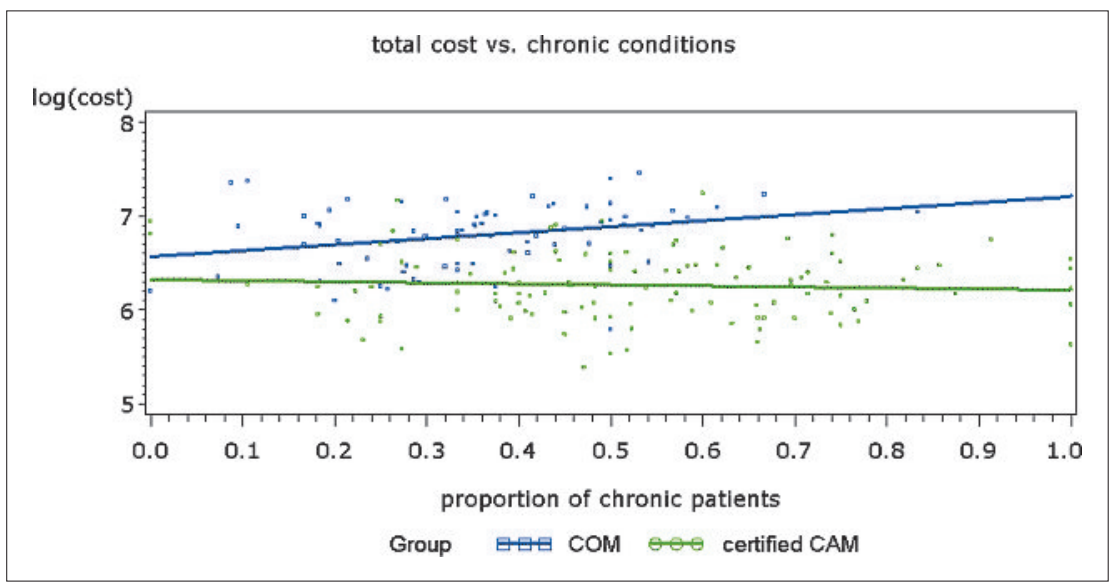

\section{Tabelle 4}

Nutzenorientierte Masszahlen, erwachsene Patienten, 2002/2003.

\begin{tabular}{|c|c|c|c|}
\hline & $\begin{array}{l}\text { Zertifizierte } \\
\text { CAM-Ärzte }\end{array}$ & $\begin{array}{l}\text { Nicht-zertifizierte } \\
\text { CAM-Ärzte }\end{array}$ & COM-Ärzte \\
\hline $\begin{array}{l}\text { Beschwerden im Vergleich zum Arzt- } \\
\text { besuch vor einem Monat nicht mehr } \\
\text { vorhanden oder wesentlich schwächer }\end{array}$ & $60 \%$ & $57 \%$ & $58 \%$ \\
\hline $\begin{array}{l}\text { Erwartungen an die Behandlung } \\
\text { vollkommen erfüllt }\end{array}$ & $34 \%$ & $34 \%$ & $33 \%$ \\
\hline $\begin{array}{l}\text { Sehr zufrieden mit dem bisherigen } \\
\text { Verlauf der Behandlung }\end{array}$ & $51 \%$ & $51 \%$ & $44 \%$ \\
\hline $\begin{array}{l}\text { Unerwünschte Nebenwirkungen } \\
\text { im Zusammenhang mit Behandlung: ja }\end{array}$ & $9 \%$ & $13 \%$ & $15 \%$ \\
\hline $\begin{array}{l}\text { Anteil Konsultationen mit } \\
\text { Bescheinigung einer Arbeitsunfähigkeit }\end{array}$ & $7 \%$ & $13 \%$ & $14 \%$ \\
\hline $\begin{array}{l}\text { Anzahl Wochen bescheinigte } \\
\text { Arbeitsunfähigkeit pro Patient, } \\
\text { bei Bescheinigung einer Arbeitsunfähigkeit a }\end{array}$ & 4,6 & 5,2 & 2,4 \\
\hline
\end{tabular}

Beziehung», «Patienteninformation und -motivation» sowie «Betreuungskontinuität und Kooperation» zugunsten der zertifizierten CAM-Ärzte aus.

Vorteile für die Komplementärmedizin, vor allem wenn sie von zertifizierten Ärzten ausgeübt wird, zeigten sich auch hinsichtlich der Nebenwirkungen der Behandlung. Uneinheitlich sind die Unterschiede demgegenüber bei den bescheinigten Arbeitsunfähigkeitstagen. Der Anteil von Konsultationen mit bescheinigten Arbeitsunfähigkeitstagen ist bei den zertifizierten CAM-Ärzten erheblich geringer als bei den nichtzertifizierten CAM-Ärzten und bei den COM-Ärzten. Falls eine Arbeitsunfähigkeit bescheinigt wurde, war diese jedoch bei den CAM-Ärzten deutlich länger als bei den COM-Ärzten, was insgesamt darauf hinweist, dass die indirekten Kosten von zertifizierten CAM-Ärzten und COM-Ärzten in etwa gleich sind. Diejenigen von nicht-zertifizierten CAM-Ärzten sind demgegenüber deutlich höher. Bei diesen beobachteten Zahlen sind allerdings Unterschiede bei den Patientenpopulationen nicht berücksichtigt.

\section{Diskussion}

Sowohl hinsichtlich der Kosten und insbesondere der Kostenstruktur als auch hinsichtlich der nutzenbezogenen Ergebnisse zeigen sich aufschlussreiche Unterschiede zwischen zertifizierter und nicht-zertifizierter Komplementärmedizin sowie konventioneller Medizin. Sie stehen im Zusammenhang mit unterschiedlichen Behandlungsansätzen und -philosophien.

\section{Unterschiedliche Kostenstruktur}

Ärzte, die nebst Schul- auch Komplementärmedizin einsetzen, generieren pro Patient gleich hohe Kosten wie ausschliesslich schulmedizinisch tätige Ärzte. Dies ist der Fall, obwohl Konsultationen bei zertifizierten CAM-Ärzten deutlich länger dauern und die Anzahl Konsultationen pro Patient etwas höher ist als bei rein schulmedizinisch tätigen COM-Ärzten. Die zertifizierten CAM-Ärzte machen jedoch die damit verbundenen höheren konsultationsbezogenen Kosten offensichtlich durch geringere veranlasste Kosten und besonders durch geringere Medikamentenkosten wett.

Vor allem in einem Gesundheitssystem mit einer hohen Ärztedichte sind allerdings nicht nur die Kosten pro Patient relevant, sondern auch die Kosten pro Arzt. Sie sind bei zertifizierten CAM-Ärzten deutlich geringer als bei COM-Ärzten. Die Kostendifferenz kommt in erster Linie dadurch zustande, dass die zertifizierten CAM-Ärzte weniger Patienten behandeln, sich dafür aber mehr Zeit nehmen, ohne dass dadurch jedoch die Kosten pro Patient höher ausfallen. Zum einen kommt dies den Patienten respektive der Qualität der Arzt-Patienten-Beziehung zugute, zum anderen wird die Problematik der hohen Ärztedichte in der Schweiz indirekt entschärft, indem durch die längeren Konsultationszeiten der zertifizierten CAM-Ärzte sozusagen Arztkapazität aus einem System mit einer tendenziellen Überversorgung genommen wird. 


\section{Kostenvergleich basiert lediglich auf zurechenbaren Kosten}

Der santésuisse-Datenpool erlaubt es nicht, dem einzelnen Arzt Kosten für externe diagnostische Untersuchungen wie CT oder MRI, Überweisungen zu anderen Ärzten oder Spitaleinweisungen zuzuordnen. Zudem ist zu berücksichtigen, dass die Kosten pro Patient nur jenen Betrag umfassen, der im Zusammenhang mit der Tätigkeit des betreffenden Arztes steht. Kosten, die der Patient unabhängig davon sonst noch zu Lasten der obligatorischen Krankenpflegeversicherung ausgelöst hat, sind nicht erfasst.

Diesbezüglich könnte zum einen argumentiert werden, dass die Therapie bei einem zertifizierten CAM-Arzt nur sehr unvollständig und allenfalls sogar unwirksam sei und in der Folge andernorts im System die Kosten umso höher ausfallen würden. Dem widerspricht zum einen der insgesamt höhere Zufriedenheitsgrad komplementärmedizinisch behandelter Patienten. Zum anderen kann im genau umgekehrten Sinn auch angeführt werden, dass aufgrund der Kombination von qualifizierter Komplementär- und Schulmedizin, wie sie ein zertifizierter CAM-Arzt praktiziert, eine umfassende Diagnostik und Therapie möglich ist, die mithilft, auch andernorts Kosten einzusparen.

Jedenfalls zeigen die PEK-Kostendaten auf Basis der vorhandenen santésuisse-Daten, dass der umfassendere Therapieansatz der CAM-Ärzte pro Patient nicht teurer zu stehen kommt als derjenige rein schulmedizinisch tätiger Ärzte. Ferner kann argumentiert werden, dass die deutlich tieferen Gesamtkosten für Medikamente bei Patienten von CAM-Ärzten mittel- und längerfristig mit geringeren unerwünschten Arzneimittelwirkungen und damit verbundenen, allenfalls auch andernorts im Gesundheitssystem anfallenden Folgekosten einhergehen.

\section{Wie objektiv und aussagekräftig sind Selbsteinschätzungen?}

Beim Kostenvergleich mit in Betracht zu ziehen ist die Tatsache, dass der Anteil chronisch Kranker vor allem bei den zertifizierten CAM-Ärzten deutlich höher ist als bei den COM-Ärzten. Als chronisch krank wurde definiert, wer während mehr als drei Monaten von einem Hauptsymptom betroffen war. Zwar könnte behauptet werden, dieses Kriterium sei unscharf, und es sei insbesondere möglich, dass CAM-Ärzte im Gegensatz zu COM-Ärzten lediglich leichtere chronische Erkrankungen behandelten.

Dem steht jedoch entgegen, dass gemäss Selbsteinschätzung der Patienten der Schweregrad ihrer Erkrankung im Fall von CAM-Patienten deutlich höher liegt als derjenige von COM-Patienten. Dem Argument, eine solche Selbsteinschätzung sei nicht objektiv, kann entgegengehalten werden, dass gemäss anderen Studien Selbsteinschätzungen durch die Patienten durchaus verlässliche Aussagekraft zukommt. Auch die Einschätzung durch die Ärzte selbst weist in diese Richtung. Ferner haben sich in anderen, kürzlich durchgeführten Studien zur Grundversorgung im Schweizerischen
Gesundheitswesen keine Unterschiede hinsichtlich des subjektiv (durch den Patienten) und des objektiv (durch den Arzt) eingeschätzten Gesundheitszustandes gezeigt.

Sich für die Patienten Zeit nehmen zahlt sich aus Der Umstand, dass sich vor allem zertifizierte CAMÄrzte deutlich mehr Zeit für ihre Patienten nehmen, stellt wohl auch einen wesentlichen Grund für deren höhere Zufriedenheit mit der Behandlung und ihren Begleitumständen dar.

Mit Bezug auf die längere Konsultationsdauer von CAM-Ärzten weisen Busato et al. denn auch darauf hin, dass diese offenbar einen stärker interpersona-len Ansatz der Grundversorgung pflegen. Wenn künftig Strategien des Empowerments der Patienten und einer patientenzentrierten Versorgung verfolgt werden sollen, könnten die Studienergebnisse als Leitlinie verwendet werden, um die Grundversorgung dahingehend zu verbessern, das übergeordnete Ziel einer hocheffektiven interpersonalen Versorgung zu erreichen. Die Entscheidung der schweizerischen Gesundheitsbehörden, die ärztliche Komplementärmedizin wieder aus der obligatorischen Krankenpflegeversicherung auszuschliessen, zeige, dass die Wichtigkeit einer patientenzentrierten Grundversorgung vom schweizerischen Gesundheitssystem noch zu wenig anerkannt werde.

Nebst längeren Konsultationszeiten und damit verbundenen intensiveren Arzt-Patienten-Kontakten dürfte aber auch die Tatsache zur höheren Patientenzufriedenheit beigetragen haben, dass komplementärmedizinische Behandlungen im Allgemeinen mit geringeren Nebenwirkungen einhergehen als rein schulmedizinische Diagnosen und Therapien.

\section{Schlussfolgerungen}

Mit der Kombination von Schul- und Komplementärmedizin praktizieren zertifizierte CAM-Ärzte offenbar eine Form der Integrativen Medizin, bei der sich die längeren Konsultationszeiten auch pro Patient nicht in höheren Kosten niederschlagen. Deswegen und weil sich des weiteren bezüglich verschiedener nutzenbezogener Kriterien Vorteile für die ärztliche Komplementärmedizin zeigten, erscheint deren Wirtschaftlichkeit aufgrund unserer Daten gegeben.

Dies ist insbesondere dann der Fall, wenn der Miteinbezug der Komplementärmedizin in die obligatorische Krankenpflegeversicherung an die Voraussetzung eines FMH-anerkannten Fähigkeitsausweises und einer entsprechenden methodenspezifischen Zusatzausbildung geknüpft wird. Zertifizierte CAM-Ärzte schnitten jedenfalls bei gleichen Kosten in Bezug auf nutzenbezogene Kriterien gegenüber den nicht-zertifizierten CAM-Ärzten besser ab.

Allerdings sollten die vorliegenden Erkenntnisse durch zusätzliche Forschung vertieft werden, vor allem mit dem Ziel, einerseits die patientenbezogenen Kosten umfassender zu erheben und andererseits die $\mathrm{Zu}$ sammenhänge mit der Art und dem Schweregrad der Erkrankung gezielter zu erfassen und auszuwerten. 
Hans-Peter Studer *, André Busato ${ }^{* * *}$

\section{Ist ärztliche Komplementärmedizin wirtschaftlich? Eine Gesamtbeurteilung von Ergebnissen des Programms Evaluation Komplementärmedizin PEK}

\section{Einleitung}

Während sechs Jahren waren fünf Methoden der ärztlichen Komplementärmedizin provisorisch im Grundleistungskatalog der obligatorischen Krankenpflegeversicherung der Schweiz enthalten. ${ }^{1}$ Im Juni 2005 entschied der damalige Bundesrat und Gesundheitsminister Pascal Couchepin, sie wieder aus dem Leistungskatalog zu streichen. Er berief sich dabei unter anderem auf die Eidgenössische Leistungskommission als beratendes Organ, welche mit qualifizierter Mehrheit zum Schluss gekommen sei, die Wirksamkeit, Zweckmässigkeit und Wirtschaftlichkeit dieser Methoden hätten nicht nachgewiesen werden können. ${ }^{2}$

Grundlage für diese Einschätzung bildete das nationale Programm zur Evaluation in der Komplementärmedizin PEK und dessen Schlussbericht. Dieser war jedoch keinesfalls zu durchwegs negativen Ergebnissen gelangt, sondern im Gegenteil auch zu eingeschränkt oder uneingeschränkt positiven Wertungen. ${ }^{3}$ Während rund vier Jahren waren in verschiedenen PEK-Teilprojekten grosse Mengen an Daten erhoben und ausgewertet worden, die auch international Beachtung fanden.

Entsprechend irritiert zeigte sich das internationale Review Board, welches das PEK-Forschungsprogramm wissenschaftlich begleitet hatte. Im Sinne einer Protestnote verfasste es eine KonsensStellungnahme, in welcher es einstimmig festhielt, „,dass die letzte, aber entscheidende Phase des PEK Prozesses vom für solche Verfahren üblichen und zu erwartenden Ablauf stark abwich.“4 Auch die Geschäftsprüfungskommissionen beider Parlamente rügten Bundesrat Couchepin in ihrem Jahresbericht 2008. ${ }^{5}$ Sie hielten in einem Brief an den Gesundheitsminister fest, es sei unverständlich, dass das Projekt „,nicht in geordneter Art und Weise abgeschlossen wurde.“6 Das behördliche Vorgehen könne den Anschein erwecken, in bestimmten Bereichen seien eher politische als objektive Erwägungen entscheidend gewesen.

Am 17. Mai 2009 stimmte die schweizerische Bevölkerung dem parlamentarischen Gegenvorschlag zur Volksinitiative „Ja zur Komplementärmedizin“ in allen Kantonen mit einer gesamthaften Ja-Mehrheit von Zweidritteln zu und verankerte damit die Komplementärmedizin in der Bundesverfassung. Mit der Abstimmungsvorlage verbunden war auch die Forderung, die ärztliche Komplementärmedizin wieder in die obligatorische Krankenpflegeversicherung aufzunehmen,

\footnotetext{
*Dr. oec., selbständiger Gesundheitsökonom, Tel. 07134438 37, hpstuder@swissonline.ch

${ }^{* * *}$ Prof. Dr. Msc., Institut für Evaluative Forschung in der Orthopädie der Universität Bern, Tel. 0316315962 , andre.busato@memcenter.unibe.ch

${ }^{1}$ Konkret handelt es sich um die Anthroposophische Medizin, die Homöopathie, die Neuraltherapie, die chinesische und die europäische Phytotherapie. Für letztere existiert allerdings bis heute kein FMH-anerkannter Fähigkeitsausweis, weshalb es phytotherapeutischen Ärzten nicht möglich war, ihre entsprechenden Leistungen explizit über die obligatorische Krankenpflegeversicherung abzurechnen. Davon ausgenommen waren und sind Arzneimittel, die auf der kassenpflichtigen Spezialitätenliste figurieren.

${ }^{2}$ Eidgenössisches Departement des Innern: Erläuterungen Entscheid komplementärmedizinische Methoden, Bern, 3. Juni 2005, Anhang, Ziff. 12. Darin zieht auch das EDI selbst den Schluss, die gesetzlichen Ansprüche an die Wirksamkeit und Zweckmässigkeit und in grossen Teilen auch diejenigen an die Wirtschaftlichkeit seien aufgrund der ihm vorliegenden Daten nicht erfüllt. Ziff. 6.

${ }^{3}$ Melchart D, Mitscherlich F, Amiet M, Eichenberger R, Koch P: Programm Evaluation Komplementärmedizin, Schlussbericht, Bern, 14. April 2005, im besonderen S. 93.

${ }^{4}$ Wallach H, Linde K, Eichenberger R, Stalder H, Kristensen FB, Kleijnen J: Konsens-Stellungnahme des PEKReview Boards zum Prozess und den Produkten von PEK, 27. September 2005.

${ }^{5}$ Geschäftsprüfungskommissionen und Geschäftsprüfungsdelegation der eidgenössischen Räte: Jahresbericht 2008, Bern, 23. Januar 2009, S. 25.

${ }^{6}$ Foppa D, Mäder Ph: Alternativmedizin: Couchepin räumt Fehler ein, in: Tages-Anzeiger, 30. Januar 2009.
} 
soweit sie den gesetzlichen Anforderungen hinsichtlich Wirksamkeit, Zweckmässigkeit und Wirtschaftlichkeit genügt. ${ }^{7}$

Um diese Forderung hinsichtlich ihrer Berechtigung beurteilen zu können, ist es naheliegend, das umfangreiche, ob der Kontroversen über den Entscheid Couchepins etwas in Vergessenheit geratene Datenmaterial des PEK-Forschungsprogramms zu Rate zu ziehen. Entsprechend ist das Ziel dieses Artikels, die PEK-Daten zur wichtigen Frage der Wirtschaftlichkeit der Komplementärmedizin in einem Gesamtkontext darzustellen und zu würdigen.

\section{Miteinbezogene Studien}

Ursprünglich bestand im Rahmen des PEK-Projekts die Absicht, in einer Teilkomponente III im Rahmen einer prospektiven, nicht-interventionellen Studie methoden- und indikationsspezifisch schulmedizinische und komplementärmedizinische Patienten ${ }^{8}$ miteinander zu vergleichen. Dabei wären auch die entsprechenden Behandlungskosten mit erhoben worden. Nachdem diese Studie jedoch von der Ethikkommission Bern nicht bewilligt worden war, musste ein anderes Vorgehen zur Beurteilung der Wirtschaftlichkeit gewählt werden. ${ }^{9}$

In der Folge stellte santésuisse, der Dachverband der Schweizer Krankenversicherer, Kostendaten für die Jahre 2002 und 2003 zur Verfügung, welche sich auf die an der PEK-Praxisstudie I beteiligten Ärzte bezogen. ${ }^{10}$ Sie beinhalteten einerseits die von den Krankenversicherern an santésuisse gemeldeten Kosten, welche ein Arzt pro Jahr in seiner Praxis generierte (konsultationsbezogene Kosten). Diese setzen sich zusammen aus den Konsultationskosten und den Kosten für direkt in der Praxis abgegebene Medikamente. Bei den Kosten, die andererseits durch den betreffenden Arzt ausserhalb seiner Praxis veranlasst wurden (veranlasste Kosten), liessen sich aufgrund der santésuisse-Datenbank nur drei Kategorien zuverlässig dem einzelnen Arzt zuordnen: Kosten für Medikamente, die auf ärztliches Rezept in einer Apotheke bezogen wurden, Kosten für externe Laboruntersuchungen und Kosten für verordnete Physiotherapien.

Diese Kostendaten erlaubten einen Vergleich zwischen den 174 rein schulmedizinisch respektive konventionell tätigen Ärzten (COM-Ärzte), die an der Praxisstudie I teilgenommen hatten, mit den 151 Ärzten, welche in ihrer Praxis auch Komplementärmedizin einsetzten, aber nicht über einen entsprechenden FMH-anerkannten Fähigkeitsausweis verfügten (nicht-zertifizierte CAMÄrzte) und den 257 Ärzten, welche zusätzlich zur Schulmedizin auch Komplementärmedizin einsetzten und hierfür über einen entsprechenden Fähigkeitsausweis verfügten (zertifizierte CAMÄrzte). ${ }^{11}$ Verglichen wurden dabei über alle drei Gruppen frei praktizierende Ärzte, die als Allgemeinmediziner oder Fachärzte für Innere Medizin in der Grundversorgung tätig waren.

Für die Beurteilung der Wirtschaftlichkeit massgebend ist neben den Kosten auch der Nutzen einer Therapiemethode. Am aussagekräftigsten sind diesbezüglich Kosten-Nutzen-Analysen, bei denen der medizinische und der soziale Nutzen ebenfalls in Geldeinheiten ausgedrückt werden. Aufgrund der Tatsache, dass die geplante Studienkomponente III nicht durchgeführt werden konnte, sind hierfür jedoch zu wenig verlässliche Daten vorhanden. Es lassen sich aber zumindest in Bezug auf eine Kosten-Wirksamkeits-Analyse Aussagen machen, aufgrund derer sich die Wirtschaftlichkeit der ärztlichen Komplementärmedizin abschätzen lässt.

Grundlage hierfür bildet zum einen die Praxisstudie II, bei der über einen Zeitraum von einem Jahr bei 318 Ärzten (71 COM Ärzte, 77 nicht-zertifizierte CAM Ärzte, 170 zertifizierte CAM Ärzte), die an der Praxisstudie I teilgenommen hatten, an vier saisonalen Stichtagen systematisch insgesamt 11'615 Behandlungen von Patienten dokumentiert wurden, die älter als 16 Jahre waren

\footnotetext{
${ }^{7}$ Vgl. die Erläuterungen des Bundesrats zur Volksabstimmung vom 17. Mai 2009, S. 9.

${ }^{8}$ Überall, wo in dieser Arbeit sprachlich die männliche Form von Substantiven verwendet wird, ist die weibliche Form mit gemeint.

${ }^{9}$ Melchart et al., 2005, S. $19 f$.

${ }^{10}$ Weil für die Jahre 2002 und 2003 nicht alle Krankenversicherer ihre Daten an santésuisse lieferten, umfasste die santésuisse-Datenbank nur rund 95\% der effektiven Kosten. Melchart et al., 2005, S. 55.

${ }^{11}$ Vgl. zur Auswahl und näheren Beschreibung dieser Stichprobe von insgesamt 582 Ärzten: Melchart et al., 2005, S. 32f, 49.
} 
(5'832 bei zertifizierten CAM-Ärzten, 2'795 bei nicht-zertifizierten CAM-Ärzten und 2'988 bei COM-Ärzten). Weiter standen Daten von 6'133 Patienten zur Verfügung, die einen Monat nach der Behandlung einen Fragebogen beantwortet hatten. Aus diesen Befragungen ergeben sich unter anderem Hinweise auf den Behandlungserfolg und die Zufriedenheit mit der Behandlung, aber auch Angaben zu den Nebenwirkungen sowie zu den mit der Erkrankung verbundenen Arbeitsausfalltagen.

Sowohl hinsichtlich der Kosten als auch des Nutzens ist immer der Vergleich mit der Schulmedizin massgebend. Die Wirtschaftlichkeit komplementärmedizinischer Methoden ist im Minimum dann gegeben, wenn sie im Vergleich zu schulmedizinischen, bereits in der obligatorischen Krankenpflegeversicherung enthaltenen Methoden keine höheren Kosten verursachen und keinen geringeren Nutzen mit sich bringen. ${ }^{12}$

\section{Ergebnisse}

\section{Kosten pro Arzt}

Der Vergleich der empirischen Daten ergab pro Arzt grosse Unterschiede bei den jährlich zulasten der obligatorischen Krankenpflegeversicherung generierten Kosten. Wie Tabelle 1 zeigt, sind die beobachteten Kosten bei den zertifizierten CAM-Ärzten durchweg am tiefsten und bei den rein schulmedizinisch tätigen COM-Ärzten durchweg am höchsten. Dazwischen liegen die Kosten bei

Tabelle 1: Jährliche Kosten (in SFr) zulasten der obligatorischen Krankenpflegeversicherung von zertifizierten und nicht-zertifizierten komplementärmedizinisch tätigen Ärzten (CAMÄrzten) sowie von rein schulmedizinisch tätigen Ärzten (COM-Ärzten), 2002/2003, ohne Kostenselbstbehalte der Patienten ${ }^{13}$

\begin{tabular}{|c|c|c|c|c|c|c|}
\hline \multirow[t]{2}{*}{ Kostenart (SFr) } & \multicolumn{2}{|c|}{ Zertifizierte CAM-Ärzte } & \multicolumn{2}{|c|}{$\begin{array}{c}\text { Nicht-zertifizierte CAM- } \\
\text { Ärzte }\end{array}$} & \multicolumn{2}{|c|}{ COM-Ärzte } \\
\hline & Mittelwert & Median & Mittelwert & Median & Mittelwert & Median \\
\hline - Konsultationskosten & $235^{\prime} 900$ & $206^{\prime} 300$ & $282 ’ 500$ & $261 ' 800$ & $302 ' 900$ & $300 ’ 400$ \\
\hline $\begin{array}{l}\text { - Direkt abgegebene } \\
\text { Medikamente }\end{array}$ & $63 ’ 200$ & $16 ’ 500$ & $156^{\prime} 600$ & $84 ’ 900$ & $156 ’ 200$ & $35^{\prime} 200$ \\
\hline $\begin{array}{l}\text { Konsultations- } \\
\text { bezogene Kosten }\end{array}$ & $298 ’ 400$ & $239 ' 700$ & 435,000 & $396 ’ 500$ & $457^{\prime} 900$ & $374 ' 700$ \\
\hline $\begin{array}{l}\text { - Verschriebene } \\
\text { Medikamente }\end{array}$ & $77^{\prime} 600$ & $31 ’ 500$ & $186 ’ 500$ & $75^{\prime} 800$ & $285^{\prime} 700$ & $223 ’ 900$ \\
\hline $\begin{array}{l}\text { - Externe Labor- } \\
\text { analysen }\end{array}$ & $19^{\prime} 400$ & $12^{\prime} 600$ & $30^{\prime} 100$ & $22 ’ 200$ & $38^{\prime} 500$ & $30 ’ 500$ \\
\hline $\begin{array}{l}\text { - Verordnete } \\
\text { Physiotherapien }\end{array}$ & $24 ' 900$ & $15^{\prime} 100$ & $399^{\prime} 300$ & $34^{\prime} 000$ & $52 ׳ 400$ & $47^{\prime} 200$ \\
\hline Veranlasste Kosten & $118 \% 800$ & $65 ’ 300$ & $255^{\prime} 100$ & $1422^{\prime} 700$ & $376{ }^{\prime} 500$ & $292 ' 800$ \\
\hline Gesamtkosten & $417 ’ 200$ & $330{ }^{\prime} 400$ & $690 ’ 100$ & $655 ’ 900$ & $834^{\prime} 400$ & $799 ' 400$ \\
\hline $\begin{array}{l}\text { - davon: Medikamen- } \\
\text { tenkosten total }\end{array}$ & $142^{\prime} 700$ & $73^{\prime} 800$ & $343^{\prime} 100$ & $303 ’ 200$ & $441 ' 400$ & $405^{\prime} 400$ \\
\hline
\end{tabular}

\footnotetext{
${ }^{12}$ Ebenfalls gegeben wäre die Wirtschaftlichkeit dann, wenn die Kosten zwar höher, aber auch der Nutzen entsprechend grösser wäre oder wenn bei einem kleineren Nutzen die Kosten entsprechend geringer wären. Falls die ärztliche Komplementärmedizin entweder hinsichtlich der Kosten oder des Nutzen gegenüber der Schulmedizin Vorteile aufweist, ist die Wirtschaftlichkeit ohnehin als gegeben zu erachten.

${ }^{13}$ Daten gerundet auf SFr 100. Differenzen bei der Addition der Mittelwerte ergeben sich daraus, dass die Zellenwerte einzeln berechnet wurden. Die Zahlen entsprechen weitgehend denjenigen, die im PEK-Schlussbericht (Melchart et al., 2005, S. 47) publiziert wurden. Sie basieren jedoch auf einer etwas grösseren Stichprobe und umfassen auch die nicht-zertifizierten komplementärmedizinisch tätigen Ärzte sowie die Medianwerte.
} 
denjenigen Ärzten, die Komplementärmedizin ohne entsprechenden Fähigkeitsausweis zusätzlich zur Schulmedizin einsetzten (nicht-zertifizierte CAM-Ärzte).

Besonders gross sind die Unterschiede bei den veranlassten Kosten, etwas weniger ausgeprägt bei den konsultationsbezogenen Kosten (inkl. direkt abgegebene Medikamente) und insbesondere bei den reinen Konsultationskosten (ohne Medikamente). Der Mittelwert der gesamten Medikamentenkosten, die sich aus den Kosten für direkt in der Praxis abgegebene Medikamente und denjenigen für verschriebene Medikamente zusammensetzen, beläuft sich bei den zertifizierten CAMÄrzten mit SFr 142'700 lediglich auf ein Drittel desjenigen der COM-Ärzte mit SFr 441'400. Was den Mittelwert der Gesamtkosten anbelangt, so ist er bei zertifizierten CAM-Ärzten mit SFr 417'200 genau halb so hoch wie bei den COM-Ärzten mit SFr 834'000. Bei den nichtzertifizierten CAM-Ärzten liegt der Mittelwert der Gesamtkosten bei SFr 690’100.

\section{Modellbasierte Kosten pro Arzt}

Beim Vergleich der Kosten pro Arzt ist jedoch zu beachten, dass die Zusammensetzung der Patientenkollektive in den jeweiligen Arztpraxen verschieden war und dass sich die Praxen auch von der Struktur her unterschieden. Entsprechend wurden die empirischen Kosten mit einem nichtparametrischen Regressionsmodell für die folgenden neun Variablen statistisch korrigiert: Ort der Praxis nach Kanton, Urbanisationsgrad des Praxisorts, Art der Praxis (Einzel- oder Gruppenpraxis), Geschlecht des Arztes, Anzahl der Jahre, in welchen der Arzt nach dem Staatsexamen berufstätig war, Anteil weiblicher Patienten, Durchschnittsalter der Patienten, Anteil Hausbesuche, Anteil unfallbezogener Konsultationen. ${ }^{14}$ Daraus resultierten pro Arztpraxis und Jahr statistisch korrigierte respektive adjustierte Kosten gemäss Tabelle 2.

Es fällt auf, dass sich die Kosten der zertifizierten CAM-Ärzte signifikant von denjenigen der nicht-zertifizierten CAM-Ärzte und der COM-Ärzte unterscheiden. Sie liegen bei allen berechneten Kostenkategorien unter denjenigen der beiden anderen Gruppen und insbesondere unter denjenigen der ausschliesslich schulmedizinisch tätigen Ärzte (COM-Ärzte). Bei den statistisch adjustierten Gesamtkosten liegen die Kosten der zertifizierten CAM-Ärzte mit SFr 531'600 um 29\%

Tabelle 2: Statistisch adjustierte jährliche Kosten (in SFr) zulasten der obligatorischen Krankenpflegeversicherung von zertifizierten und nicht-zertifizierten komplementärmedizinisch tätigen Ärzten (CAM-Ärzten) sowie von rein schulmedizinisch tätigen Ärzten (COM-Ärzten), 2002/2003, ohne Kostenselbstbehalte der Patienten ${ }^{15}$

\begin{tabular}{|c|c|c|c|c|c|c|c|}
\hline \multirow[t]{2}{*}{ Kostenart (SFr) } & \multicolumn{2}{|c|}{ Zertifizierte CAM-Ärzte } & \multicolumn{2}{|c|}{$\begin{array}{c}\text { Nicht-zertifizierte } \\
\text { CAM-Ärzte }\end{array}$} & \multicolumn{2}{|c|}{ COM-Ärzte } & \multirow[t]{2}{*}{$r^{23}$} \\
\hline & LS-mean ${ }^{l}$ & $\mathrm{SE}^{2}$ & LS-mean & SE & LS-mean & SE & \\
\hline $\begin{array}{l}\text { Konsultations- } \\
\text { bezogene Kosten }\end{array}$ & $386^{\prime} 100^{a}$ & $\pm 37^{\prime} 400$ & $428^{\prime} 400^{b}$ & $\pm 37^{\prime} 900$ & $436^{\prime} 800^{b}$ & $\pm 38^{\prime} 400$ & $39.5 \%$ \\
\hline $\begin{array}{l}\text { Veranlasste } \\
\text { Kosten }\end{array}$ & $154^{\prime} 900^{a}$ & $\pm 28 ’ 300$ & $228^{\prime} 400^{b}$ & $\pm 28^{\prime} 900$ & $301^{\prime} 600^{b}$ & $\pm 29 ’ 000$ & $47.7 \%$ \\
\hline Gesamtkosten & $531^{\prime} 600^{a}$ & $\pm 48 ' 500$ & $664^{\prime} 200^{b}$ & $\pm 49 ’ 600$ & $748^{\prime} 700^{b}$ & $\pm 50 ’ 100$ & $42.8 \%$ \\
\hline $\begin{array}{l}\text { davon: Medika- } \\
\text { mentenkosten }\end{array}$ & $229^{\prime} 900^{a}$ & $\pm 35^{\prime} 500$ & $309^{\prime} 000^{b}$ & $\pm 36 ’ 300$ & $373^{\prime} 900^{b}$ & $\pm 36^{\prime} 000$ & $40.5 \%$ \\
\hline
\end{tabular}

a,b Unterschiedliche Buchstaben weisen auf signifikante Unterschiede zwischen den Gruppen hin $(\mathrm{p}<0.05)$.

${ }^{1}$ Modellbasierter Mittelwert, errechnet mittels nicht-parametrischer Regression

${ }^{2}$ Standardfehler

${ }^{3} \mathrm{r}^{2}=$ Anteil der durch das statistische Modell erklärten Varianz der Zielvariablen

\footnotetext{
${ }^{14} \mathrm{Vgl}$. Busato A, Eichenberger R, Kuenzi B: Extent and structure of health insurance expenditures for complementary and alternative medicine in Swiss primary care. BMC Health Services Research 2006, 6:132.

${ }^{15}$ Daten gerundet auf SFr 100. Differenzen bei der Addition der Mittelwerte ergeben sich daraus, dass die Zellenwerte einzeln berechnet wurden.
} 
tiefer als diejenigen der COM-Ärzte mit SFr $748^{\prime} 700$ pro Praxis und Jahr. Besonders gross sind die Unterschiede bei den veranlassten Kosten, etwas geringer bei den konsultationsbezogenen Kosten. Werden die Medikamentenkosten separat ausgewiesen, so liegen sie bei den zertifizierten CAM-Ärzten mit SFr 229'900 um 38.5\% tiefer als bei den COM-Ärzten mit SFr 373'900. Mit einem $r^{2}$ zwischen 39.5 und 47.7 wird dabei jeweils ein relativ hoher Anteil der Varianz der Zielvariablen erklärt.

\section{Kosten pro Patient}

Für einen Kostenvergleich zwischen Komplementärmedizin und Schulmedizin sind indes nicht nur die Kosten pro Arztpraxis relevant, sondern auch die Kosten pro Patient. Sie errechnen sich aus den Kosten pro Arzt dividiert durch die Anzahl der in den Jahren 2002 und 2003 behandelten Patienten. Diese war in den drei Gruppen sehr unterschiedlich: ein zertifizierter CAM-Arzt behandelte im Durchschnitt pro Jahr deutlich weniger - nämlich 648 - Patienten als ein nichtzertifizierter CAM-Arzt (946 Patienten) oder ein COM-Arzt (988 Patienten). ${ }^{16}$

Der Hauptgrund dafür war, dass die zertifizierter CAM-Ärzte pro Konsultation erheblich mehr Zeit aufwendeten, nämlich durchschnittlich 29.1 Minuten, verglichen mit nicht-zertifizierten CAM-Ärzten mit 21.1 Minuten und COM-Ärzten mit 17.1 Minuten durchschnittlicher Konsultationsdauer. Zudem liegt die ausgewiesene Anzahl Konsultationen pro Patient und Jahr bei zertifizierten CAM-Ärzten mit 4.7 etwas höher als bei nicht-zertifizierten CAM-Ärzten und bei COMÄrzten mit je 4.2 Konsultationen pro Patient und Jahr. ${ }^{17}$

Tabelle 3: Kosten pro Patient (in SFr) zulasten der obligatorischen Krankenpflegeversicherung bei zertifizierten und nicht-zertifizierten komplementärmedizinisch tätigen Ärzten (CAMÄrzten) sowie bei rein schulmedizinisch tätigen Ärzten (COM-Ärzten), 2002/2003, ohne Kostenselbstbehalte der Patienten ${ }^{18}$

\begin{tabular}{|c|c|c|c|c|c|c|}
\hline \multirow[t]{2}{*}{ Kostenart (SFr) } & \multicolumn{2}{|c|}{ Zertifizierte CAM-Ärzte } & \multicolumn{2}{|c|}{$\begin{array}{l}\text { Nicht-zertifizierte CAM- } \\
\text { Ärzte }\end{array}$} & \multicolumn{2}{|c|}{ COM-Ärzte } \\
\hline & Mittelwert & Median & Mittelwert & Median & Mittelwert & Median \\
\hline - Konsultationskosten & 445 & 364 & 325 & 306 & 335 & 313 \\
\hline $\begin{array}{l}\text { - Direkt abgegebene } \\
\text { Medikamente }\end{array}$ & 73 & 30 & 150 & 83 & 139 & 46 \\
\hline $\begin{array}{l}\text { Konsultations- } \\
\text { bezogene Kosten }\end{array}$ & 517 & 434 & 474 & 452 & 473 & 451 \\
\hline $\begin{array}{l}\text { - Verschriebene } \\
\text { Medikamente }\end{array}$ & 127 & 58 & 226 & 116 & 359 & 279 \\
\hline $\begin{array}{l}\text { - Externe Labor- } \\
\text { analysen }\end{array}$ & 31 & 22 & 38 & 27 & 45 & 33 \\
\hline $\begin{array}{l}\text { - Verordnete } \\
\text { Physiotherapien } \\
\end{array}$ & 35 & 27 & 49 & 41 & 56 & 54 \\
\hline Veranlasste Kosten & 190 & 119 & 312 & 216 & 458 & 362 \\
\hline Gesamtkosten & 708 & 628 & 786 & 747 & 931 & 869 \\
\hline $\begin{array}{l}\text { - davon: Medikamen- } \\
\text { tenkosten total }\end{array}$ & 197 & 142 & 377 & 349 & 481 & 453 \\
\hline
\end{tabular}

\footnotetext{
${ }^{16}$ Melchart et al., 2005, S. 50.

${ }^{17}$ Melchart et al., 2005, S. 50. Es wurde hier bewusst auf die empirischen Werte abgestellt, weil sich daraus die zeitliche Limitierung, bezogen auf die Anzahl behandelter Patienten pro Jahr, ergibt. Der unterschiedlichen Zusammensetzung der Patientenkollektive wird bei der statistischen Adjustierung der Kosten Rechnung getragen.

${ }^{18}$ Melchart et al., 2005, S. 50, ergänzt durch Medianwerte und Zahlen zu Unterkategorien der konsultationsbezogenen und der veranlassten Kosten. Differenzen bei der Addition der Mittelwerte ergeben sich daraus, dass die Zellenwerte einzeln berechnet wurden.
} 
Wie Tabelle 3 zeigt, schlägt sich die Tatsache, dass Konsultationen bei zertifizierten CAM-Ärzten deutlich länger dauern und pro Patient etwas häufiger stattfinden als bei nicht-zertifizierten CAMÄrzten und bei COM-Ärzten, darin nieder, dass bei ersteren die beobachteten Konsultationskosten pro Patient und Jahr höher sind. Im Gegensatz dazu sind jedoch die veranlassten Kosten und insbesondere die Medikamentenkosten im Vergleich deutlich tiefer, was die höheren Konsultationskosten mehr als kompensiert. Entsprechend liegen die patientenbezogenen Gesamtkosten mit SFr 708 pro Jahr bei den zertifizierten CAM-Ärzten am tiefsten und bei den COM-Ärzten mit SFr 931 am höchsten. Wiederum dazwischen liegen die nicht-zertifizierten CAM-Ärzte mit SFr 786 pro Patient und Jahr.

\section{Modellbasierte Kosten pro Patient}

Um den Unterschieden in der Zusammensetzung der Patientenkollektive und in der Struktur der Praxis Rechnung zu tragen, wurden auch die empirischen Kosten pro Patient und Jahr mit einem nicht-parametrischen Regressionsmodell statistisch adjustiert. Dabei wurden dieselben Variablen miteinbezogen wie bei der statistischen Korrektur der Kosten pro Arzt.

Wie Tabelle 4 zeigt, ergaben sich in der Folge signifikant höhere konsultationsbezogene Kosten pro Patient der zertifizierten CAM-Ärzte (SFr 452) im Vergleich zu den nicht-zertifizierten CAMÄrzten (SFr 437) und den COM-Ärzten (SFr 403). Auch die Unterschiede der konsultationsbezogenen Kosten von nicht-zertifizierten CAM-Ärzten und von COM-Ärzten waren signifikant. Umkehrt waren die veranlassten Kosten bei den zertifizierten CAM-Ärzten (SFr 191) signifikant tiefer als diejenigen der nicht-zertifizierten CAM-Ärzte (SFr 245) und der COM-Ärzte (SFr 280).

Diese beiden gegenläufigen Tendenzen heben sich bei den Gesamtkosten auf. Sie sind zwar mit SFr 661 bei den zertifizierten CAM-Ärzten am tiefsten, gefolgt von denjenigen der COM-Ärzte mit SFr 709 und jenen der nicht-zertifizierten CAM-Ärzte mit SFr 730. Die Unterschiede sind jedoch statistisch nicht signifikant, das heisst die patientenbezogenen Kosten der drei Ärztegruppen sind bei der gegebenen Stichprobengrösse hinsichtlich der miteinbezogenen Kostenkategorien gleich.

Ebenfalls signifikante Kostenunterschiede ergeben sich dann, wenn die Medikamentenkosten pro Patient separat ausgewiesen werden. Sie sind bei den zertifizierten CAM-Ärzten mit SFr 254

Tabelle 4: Statistisch adjustierte jährliche Kosten pro Patient (in SFr) zulasten der obligatorischen Krankenpflegeversicherung von zertifizierten und nicht-zertifizierten komplementärmedizinisch tätigen Ärzten (CAM-Ärzten) sowie von rein schulmedizinisch tätigen Ärzten (COMÄrzten), 2002/2003, ohne Kostenselbstbehalte der Patienten ${ }^{19}$

\begin{tabular}{|c|c|c|c|c|c|c|c|}
\hline \multirow[t]{2}{*}{ Kostenart (SFr) } & \multicolumn{2}{|c|}{$\begin{array}{c}\text { Zertifizierte CAM- } \\
\text { Ärzte }\end{array}$} & \multicolumn{2}{|c|}{$\begin{array}{c}\text { Nicht-zertifizierte CAM- } \\
\text { Ärzte }\end{array}$} & \multicolumn{2}{|c|}{ COM-Ärzte } & \multirow[t]{2}{*}{$r^{23}$} \\
\hline & $L S$-mean ${ }^{I}$ & $\mathrm{SE}^{2}$ & LS-mean & SE & LS-mean & SE & \\
\hline $\begin{array}{l}\text { Konsultations- } \\
\text { bezogene Kosten }\end{array}$ & $452^{a}$ & \pm 44 & $437^{a b}$ & \pm 44 & $403^{b}$ & \pm 44 & $39.5 \%$ \\
\hline $\begin{array}{l}\text { Veranlasste } \\
\text { Kosten }\end{array}$ & $191^{a}$ & \pm 57 & $245^{b}$ & \pm 58 & $280^{b}$ & \pm 58 & $54.2 \%$ \\
\hline Gesamtkosten & 661 & $\pm \mathbf{5 7}$ & 730 & $\pm \mathbf{5 7}$ & 709 & \pm 58 & $55.5 \%$ \\
\hline $\begin{array}{l}\text { davon: Medika- } \\
\text { mentenkosten }\end{array}$ & $254^{a}$ & \pm 48 & $330^{b}$ & \pm 48 & $333^{b}$ & \pm 48 & $65.5 \%$ \\
\hline
\end{tabular}

a,b Unterschiedliche Buchstaben weisen auf signifikante Unterschiede zwischen den Gruppen hin $(\mathrm{p}<0.05)$.

${ }^{1}$ Modellbasierter Mittelwert, errechnet mittels nicht-parametrischer Regression

${ }^{2}$ Standardfehler

${ }^{3} \mathrm{r}^{2}=$ Anteil der durch das statistische Modell erklärten Varianz der Zielvariablen

\footnotetext{
${ }^{19}$ Melchart et al., 2005, S. 50, ergänzt durch Standardfehler und der durch das statistische Modell erklärten Varianz der Zielvariablen $\left(\mathrm{r}^{2}\right)$. Differenzen bei der Addition der Mittelwerte ergeben sich daraus, dass die Zellenwerte einzeln berechnet wurden.
} 
signifikant tiefer als diejenigen der nicht-zertifizierten CAM-Ärzte mit SFr 330 und diejenigen der COM-Ärzte mit SFr 333. Mit einem $r^{2}$ zwischen 39.5 und 65.5 wird dabei wiederum ein jeweils relativ hoher Anteil der Varianz der Zielvariablen erklärt.

\section{Unterschiedlicher Gesundheitszustand der Patienten}

In die statistische Korrektur der Kostendaten bewusst nicht miteinbezogen wurde der unterschiedliche Gesundheitszustand der Patienten. Einerseits wurde er als intrinsische Komponente des Angebots und der Nachfrage innerhalb einer spezifischen Behandlungsphilosophie erachtet. Das heisst, Patienten haben spezifische Diagnose- und Behandlungsprozeduren vor Augen, gemäss derer sie einen bestimmten Arzt auswählen. ${ }^{20}$ So behandeln COM-Ärzte und auch nichtzertifizierte CAM-Ärzte zum Beispiel häufiger kardiovaskuläre Erkrankungen, Stoffwechselbeschwerden und äussere Verletzungen, zertifizierte CAM-Ärzte jedoch mehr Patienten mit psychiatrischen Beschwerden oder mit Krankheiten des Nervensystems sowie mehr Patienten mit muskoskeletalen Rücken- und Gelenkbeschwerden oder mit Hauterkrankungen. ${ }^{21}$

Andererseits ist der Gesundheitszustand der Patienten auf Niveau Praxis schwierig zu bestimmen. Er ergibt sich unter anderem aus der Selbsteinschätzung der Patienten und der Einschätzung der behandelnden Ärzte, aus der Anzahl und dem Schweregrad von Komorbiditäten sowie aus dem Anteil chronischer Erkrankungen.

Wie Tabelle 5 zeigt, ist der Schweregrad des Hauptsymptoms sowohl in der eigenen Einschätzung der Patienten als auch in derjenigen der behandelnden Ärzte bei den Patienten der zertifizierten CAM-Ärzte am grössten und bei den COM-Ärzten deutlich geringer. Dazwischen liegen die Werte für die Patienten der nicht-zertifizierten CAM-Ärzte. Umgekehrt ist der mit dem CharlsonKomorbiditäts-Index gemessene Anteil der lebensbedrohlichen Erkrankungen bei Patienten von COM-Ärzten am grössten und bei den zertifizierten CAM-Ärzten am geringsten. Wiederum dazwischen liegt er bei Patienten von nicht-zertifizierten CAM-Ärzten. Ausgeprägt sind die Unterschiede auch hinsichtlich des Anteils von Patienten mit chronischen Erkrankungen. Er liegt bei den zertifizierten CAM-Ärzten deutlich höher als derjenige bei den nicht-zertifizierten CAMÄrzten und den COM-Ärzten.

Tabelle 5: Schweregrad der Erkrankung, erwachsene Patienten, 2002/2003

\begin{tabular}{lccc}
\hline & $\begin{array}{c}\text { Zertifizierte CAM- } \\
\text { Ärzte }\end{array}$ & $\begin{array}{c}\text { Nicht-zertifizierte } \\
\text { CAM-Ärzte }\end{array}$ & COM-Ärzte \\
\hline $\begin{array}{l}\text { Anteil schwerwiegender Gesundheitsprob- } \\
\text { leme (Hauptsymptom), Selbsteinschätzung } \\
\text { Patient }\end{array}$ & $26 \%$ & $22 \%$ & $20 \%$ \\
$\begin{array}{l}\text { Anteil schwerwiegender Gesundheitsprob- } \\
\text { leme (Hauptsymptom), Einschätzung Arzt }\end{array}$ & $18 \%$ & $13 \%$ & $10 \%$ \\
$\begin{array}{l}\text { Anteil lebensbedrohlicher Erkrankungen } \\
\text { (Charlson-Komorbiditäts-Index }>0)\end{array}$ & $6 \%$ & $9 \%$ & $11 \%$ \\
$\begin{array}{l}\text { Anteil chronischer Erkrankungen } \\
(>3 \text { Monate) }\end{array}$ & $62 \%$ & $47 \%$ & $46 \%$ \\
\hline
\end{tabular}

Je schwerer die Erkrankung eines Patienten ist, desto höher sind in der Regel die damit verbundenen Gesundheitskosten. Was den Schweregrad anbelangt, so ergibt sich ein unterschiedliches Bild, je nach dem, ob man die Selbsteinschätzung des Patienten und diejenige des Arztes oder die Lebensbedrohlichkeit einer Erkrankung zugrunde legt. Es lässt sich also aufgrund der vorliegen-

\footnotetext{
${ }^{20}$ Busato, Eichenberger, Kuenzi, 2006, S. 8.

${ }^{21}$ Busato A, Dönges A, Herren S, Widmer M, Marian F: Health status and health care utilisation of patients in complementary and conventional primary care in Switzerland - an observational study. Family practice, VOL: 23(1), 2006, S. 116ff. Melchart et al. 2005, S. 38.
} 
den Daten und Analysen keine klare Aussage darüber machen, wie sich die mit dem Schweregrad einer Erkrankung verbundenen Kosten in den drei untersuchten Gruppen verhalten.

Was jedoch den Anteil chronisch Kranker anbelangt, so unterscheiden sich - wie Graphik 1 zeigt - die statistisch adjustierten Kosten von zertifizierten CAM-Ärzten deutlich von denjenigen von COM-Ärzten. Je grösser der Anteil chronisch Kranker bei COM-Ärzten ist, desto höher sind ihre Kosten. Bei den zertifizierten CAM-Ärzten ist dies nicht der Fall, im Gegenteil, die Kosten sinken hier mit zunehmendem Anteil chronisch Kranker sogar leicht. Zudem sind bei ihnen die chronizitätsbezogenen Kosten durchwegs deutlich tiefer als bei COM-Ärzten.

Grafik 1: Statistisch adjustierte Kosten in Abhängigkeit vom Anteil chronischer Patienten, Vergleich COM-Ärzte und zertifizierte CAM-Ärzte, logarithmische Skala, 2002/2003

total cost vs. chronic conditions

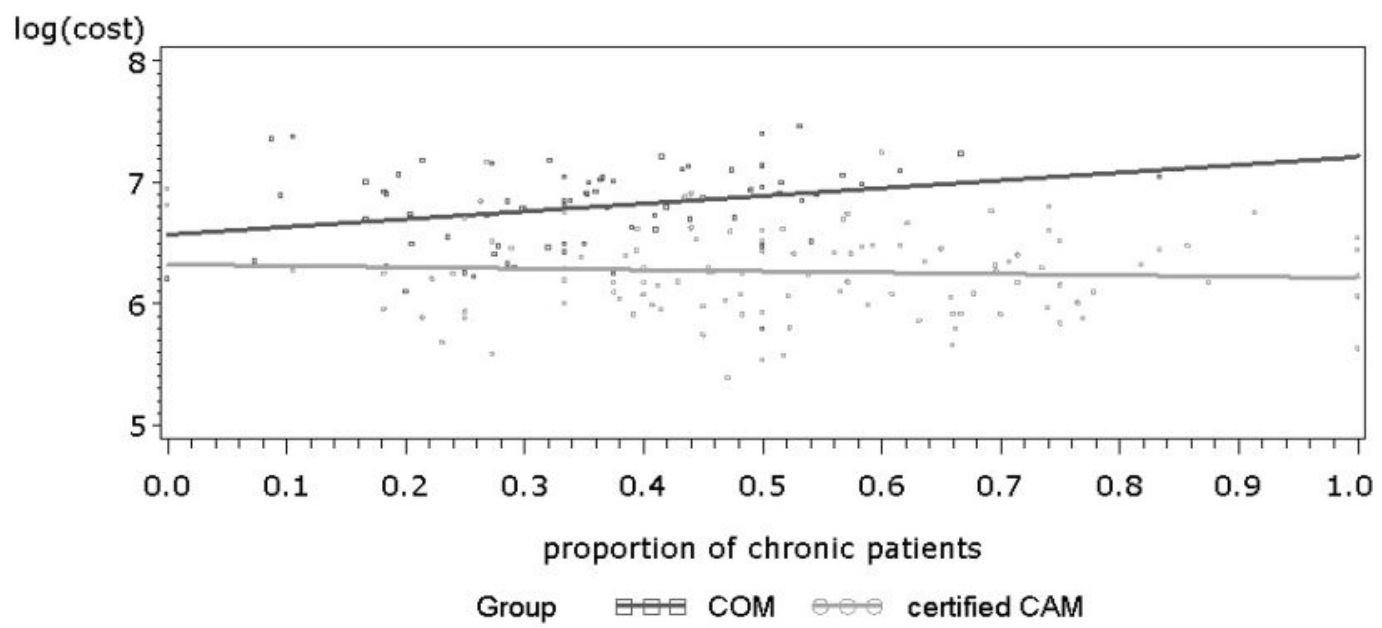

\section{Nutzenbezogene Ergebnisse}

Für die Beurteilung der Wirtschaftlichkeit der ärztlichen Komplementärmedizin relevant sind nicht nur die Kosten, sondern auch der Nutzen. Zwar ist es aufgrund der vorhandenen Daten nicht möglich, einen aussagekräftigen direkten rechnerischen Bezug zwischen diesen beiden Grössen herzustellen. Es lassen sich jedoch aufgrund der PEK-Daten Aussagen zur Zweckmässigkeit der Behandlung, zur Zufriedenheit der Patienten, zu den Nebenwirkungen sowie zu den bescheinigten Arbeitsunfähigkeitstagen machen.

Wie Tabelle 6 zeigt, ist der Anteil der Patienten, die einen Monat nach der dokumentierten Behandlung angeben, ihre Beschwerden seien nicht mehr vorhanden oder wesentlich schwächer, in allen drei untersuchten Gruppen in etwa gleich hoch. Das Gleiche gilt für die vollkommene Erfüllung der Erwartungen an die Behandlung. Sie ist in allen drei Gruppen annähernd gleich gross. Die Patienten von zertifizierten und diejenigen von nicht-zertifizierten CAM-Ärzten sind jedoch mit je 51\% in höherem Masse sehr zufrieden mit dem bisherigen Verlauf der Behandlung als die Patienten von COM-Ärzten, wo der entsprechende Prozentsatz lediglich $44 \%$ beträgt. $^{22}$

Entsprechend ergab sich auch bei der Beurteilung der Qualität der Beziehung zwischen Arzt und Patient, die mit dem Europep-Fragebogen ermittelt wurde, ein Bild, das zugunsten der CAMÄrzte ausfällt. Besonders ausgeprägt gilt dies für den Bereich „Kommunikation und Arzt-Patienten-Beziehung“, aber auch für die Bereiche „Patienteninformation und -motivation“ und „Betreuungskontinuität und Kooperation“, wo die positivste Ausprägung „,ausgezeichnet“" von den Patienten der zertifizierten CAM-Ärzte klar häufiger gewählt wurde als von jenen der COM-Ärzte. ${ }^{23}$

\footnotetext{
${ }^{22}$ Vgl. auch Busato A, Künzi B: Differences in the quality of interpersonal care in complementary and conventional medicine, im Januar 2010 zur Publikation eingereicht, BMC Complementary and Alternative Medicine.

${ }^{23}$ Melchart et al., 2005, S. 41 f.
} 
Tabelle 6: Nutzenorientierte Masszahlen, erwachsene Patienten, 2002/2003

\begin{tabular}{lccc}
\hline & $\begin{array}{c}\text { Zertifizierte CAM- } \\
\text { Ärzte }\end{array}$ & $\begin{array}{c}\text { Nicht-zertifizierte } \\
\text { CAM-Ärzte }\end{array}$ & COM-Ärzte \\
\hline $\begin{array}{l}\text { Beschwerden im Vergleich zum Arztbe- } \\
\text { such vor einem Monat nicht mehr vorhan- } \\
\text { den oder wesentlich schwächer }\end{array}$ & $60 \%$ & $57 \%$ & $58 \%$ \\
$\begin{array}{l}\text { Erwartungen an die Behandlung vollkom- } \\
\text { men erfüllt }\end{array}$ & $34 \%$ & $34 \%$ & $33 \%$ \\
$\begin{array}{l}\text { Sehr zufrieden mit dem bisherigen Verlauf } \\
\text { der Behandlung }\end{array}$ & $51 \%$ & $51 \%$ & $44 \%$ \\
$\begin{array}{l}\text { Unerwünschte Nebenwirkungen im Zu- } \\
\text { sammenhang mit Behandlung: ja }\end{array}$ & $9 \%$ & $13 \%$ & $15 \%$ \\
$\begin{array}{l}\text { Anteil Konsultationen mit Bescheinigung } \\
\text { einer Arbeitsunfähigkeit }\end{array}$ & $7 \%$ & $13 \%$ & $14 \%$ \\
$\begin{array}{l}\text { Anzahl Wochen bescheinigte Arbeitsunfä- } \\
\text { higkeit pro Patient, bei Bescheinigung } \\
\text { einer Arbeitsunfähigkeit }\end{array}$ & 4.6 & 5,2 & 2.4 \\
\hline
\end{tabular}

${ }^{a}$ nur Patienten im Alter von 17-65 Jahren

Vorteile für die Komplementärmedizin, vor allem wenn sie von zertifizierten Ärzten ausgeübt wird, zeigten sich des Weiteren hinsichtlich Nebenwirkungen der Behandlung. Wie in Tabelle 6 ersichtlich, gaben lediglich 9\% der Patienten von zertifizierten CAM-Ärzten an, die Behandlung habe zu unerwünschten Nebenwirkungen geführt, gegenüber $13 \%$ bei den nicht-zertifizierten CAM-Ärzten und $15 \%$ bei den COM-Ärzten.

Deutlich, aber uneinheitlich sind die Unterschiede zudem bei den bescheinigten Arbeitsunfähigkeitstagen. Der Anteil von Konsultationen mit bescheinigten Arbeitsunfähigkeitstagen ist bei den zertifizierten CAM-Ärzten mit 7\% erheblich geringer als bei den nicht-zertifizierten CAM-Ärzten mit $13 \%$ und bei den COM-Ärzten mit 14\%. Falls eine Arbeitsunfähigkeit bescheinigt wurde, waren es jedoch bei den zertifizierten CAM-Ärzten pro Patient im Durchschnitt 4.6 Wochen, bei den nicht-zertifizierten CAM-Ärzten 5.2 Wochen und bei den COM-Ärzten 2.4 Wochen, was insgesamt darauf hinweist, dass die indirekten Kosten von zertifizierten CAM-Ärzten und COMÄrzten in etwa gleich sind. Diejenigen von nicht-zertifizierten CAM-Ärzten sind demgegenüber deutlich höher.

Bei diesen beobachteten Zahlen sind allerdings Unterschiede bei den Patientenpopulationen nicht berücksichtigt, insbesondere der Umstand, dass in zertifizierten CAM-Praxen mehr jüngere und mehr weibliche, aber auch mehr chronisch kranke Patienten behandelt werden. Im Weiteren bleibt unklar, inwieweit sich der unterschiedliche Status von Komorbiditäten auf die indirekten Kosten auswirkt.

\section{Diskussion}

Sowohl hinsichtlich der Kosten und insbesondere der Kostenstruktur als auch hinsichtlich der nutzenbezogenen Ergebnisse zeigen sich aufschlussreiche Unterschiede zwischen zertifizierter und nicht-zertifizierter Komplementärmedizin sowie Schulmedizin respektive konventioneller Medizin. Sie stehen im Zusammenhang mit unterschiedlichen Behandlungsansätzen und -philosophien.

\section{Unterschiedliche Kostenstruktur}

Beim Kostenvergleich zwischen Schul- und Komplementärmedizin stehen die Kosten pro Patient im Zentrum. Diesbezüglich zeigten sich hinsichtlich der Gesamtsumme der analysierten Kosten- 
kategorien keine statistisch signifikanten Unterschiede, das heisst Ärzte, die nebst Schul- auch Komplementärmedizin einsetzen, generieren pro Patient gleich hohe Kosten wie ausschliesslich schulmedizinisch tätige Ärzte. ${ }^{24}$ Dies ist der Fall, obwohl Konsultationen bei zertifizierten CAMÄrzten deutlich länger dauern und die Anzahl Konsultationen pro Patient etwas höher ist als bei den rein schulmedizinisch tätigen COM-Ärzten. Die zertifizierten CAM-Ärzte machen jedoch die damit verbundenen höheren konsultationsbezogenen Kosten offensichtlich durch geringere veranlasste Kosten und im Besonderen durch geringere Medikamentenkosten wett. ${ }^{25}$

Vor allem in einem Gesundheitssystem mit einer hohen Ärztedichte relevant sind allerdings nicht nur die Kosten pro Patient, sondern auch die Kosten pro Arzt. Sie sind bei zertifizierten CAMÄrzten um 29\% geringer als bei COM-Ärzten, und das bei einem Beschäftigungsgrad, der gemessen an der verrechneten jährlichen Arbeitszeit tendenziell höher ist.

Diese Kostendifferenz kommt in erster Linie dadurch zustande, dass die zertifizierten CAM-Ärzte weniger Patienten behandeln, sich dafür aber mehr Zeit nehmen, ohne dass dadurch jedoch die Kosten pro Patient höher ausfallen. ${ }^{26}$ Zum einen kommt dies den Patienten respektive der Qualität der Arzt-Patienten-Beziehung zugute, zum anderen wird die Problematik der hohen Ärztedichte in der Schweiz indirekt entschärft, indem durch die längeren Konsultationszeiten der zertifizierten CAM-Ärzte sozusagen Arztkapazität aus einem System mit einer tendenziellen Überversorgung genommen wird. ${ }^{27}$

Letzteres gilt umso mehr, als zertifizierte CAM-Ärzte im Rahmen der komplementärmedizinischen Methoden, die sie einsetzen, zum Teil auch spezialärztliche Funktionen ausüben. Der selbstdeklarierte Anteil an Grundversorgerleistungen ist jedenfalls bei zertifizierten CAM-Ärzten mit $36.8 \%$ weniger als halb so gross wie bei COM-Ärzten mit $77.4 \%{ }^{28}$ Gleichzeitig relativiert dies das Ergebnis des Kostenvergleichs zugunsten der Komplementärmedizin, weil die von den zertifizierten CAM-Ärzten generierten Kosten nur mit jenen von schulmedizinisch tätigen Grundversorgern, nicht aber auch mit jenen von rein schulmedizinischen Spezialärzten verglichen wurden. Letztere generieren im Allgemeinen eher höhere Kosten zulasten der obligatorischen Krankenpflegeversicherung.

\section{Kostenvergleich basiert lediglich auf zurechenbaren Kosten}

Was es beim Vergleich der Kosten zertifizierter CAM-Ärzte mit denjenigen von COM-Ärzten ebenfalls zu beachten gilt, ist die Tatsache, dass er nur auf denjenigen Kostenkategorien basiert, welche im santésuisse-Datenpool zuverlässig dem einzelnen Arzt zugeordnet werden konnten. Nicht enthalten sind zum Beispiel (kostenträchtige) externe diagnostische Untersuchungen wie CT oder MRI, Überweisungen zu anderen Ärzten oder Spitaleinweisungen. ${ }^{29}$ Zudem ist zu berücksichtigen, dass die Kosten pro Patient nur jenen Betrag umfassen, welcher im Zusammenhang mit der Tätigkeit des betreffenden Arztes steht. Kosten, die der Patient unabhängig davon sonst noch zulasten der obligatorischen Krankenpflegeversicherung ausgelöst hat, sind nicht erfasst.

Diesbezüglich könnte zum einen argumentiert werden, dass die Therapie bei einem zertifizierten CAM-Arzt nur sehr unvollständig und allenfalls sogar unwirksam sei und in der Folge andernorts im System die Kosten umso höher ausfallen würden. Dem widerspricht zum einen der insgesamt höhere Zufriedenheitsgrad komplementärmedizinisch behandelter Patienten. Zum anderen kann im genau umgekehrten Sinn auch ins Feld geführt werden, dass aufgrund der Kombination von qualifizierter Komplementär- und Schulmedizin, wie sie ein zertifizierter CAM-Arzt praktiziert,

\footnotetext{
${ }^{24}$ Dies wurde auch durch die Studie von Busato et al. bestätigt, deren Daten sich lediglich auf das Jahr 2003 bezogen, bei der die Stichprobengrösse leicht angepasst und bei der für die statistische Korrektur der Daten ein vereinfachtes Verfahren verwendet wurde. Busato et al., 2006.

${ }^{25}$ Melchart et al., 2005, S. 49f.

${ }^{26}$ Es könnte auch argumentiert werden, dass zertifizierte CAM-Ärzte nur wenige Patienten haben und sie deshalb länger behandeln. Dagegen spricht, dass weit mehr zertifizierte CAM-Ärzte Wartelisten für Patienten führen als COM-Ärzte. Vgl. Melchart et al., 2005, S. 33f.

${ }^{27}$ Entsprechend wird in der schweizerischen Politik schon seit Jahren die bedingte Aufhebung des Vertragszwangs angestrebt.

${ }^{28}$ Wapf V, Busato A: Patients' motives for choosing a physician: comparison between conventional and complementary medicine in Swiss primary care, BMC Complementary and Alternative Medicine 2007, 7:41, S. 8.

${ }^{29}$ Darauf weisen auch Busato et al. in ihrer Analyse der PEK-Kostendaten hin. Busato et al., 2006, S. 10.
} 
eine umfassende Diagnostik und Therapie möglich ist, die mithilft, auch andernorts Kosten einzusparen.

Busato et al. stellten in ihrer Auswertung der PEK-Kostendaten fest, dass komplementärmedizinische Patienten ein breiteres Spektrum von medizinischen Leistungen mit grösserer Häufigkeit in Anspruch nehmen, was bezweifeln lasse, dass ein Miteinbezug der Komplementärmedizin in die obligatorische Krankenpflegeversicherung kostenneutral wäre. ${ }^{30}$ Anderseits zeigen die PEKKostendaten auf Basis der vorhandenen santésuisse-Daten, dass der umfassendere Therapieansatz der CAM-Ärzte pro Patient nicht teurer zu stehen kommt als derjenige rein schulmedizinisch tätiger Ärzte. Ferner kann argumentiert werden, dass die deutlich tieferen Gesamtkosten für Medikamente bei Patienten von CAM-Ärzten mittel- und längerfristig mit geringeren unerwünschten Arzneimittelwirkungen und damit verbundenen, allenfalls auch andernorts im Gesundheitssystem anfallenden Folgekosten einhergehen. ${ }^{31}$

\section{Substitution von anderen Leistungen durch die Komplementärmedizin}

Im Rahmen der Auswertung der Schweizerischen Gesundheitsbefragung 2002 wurde die Nutzung komplementärmedizinischer Methoden untersucht. ${ }^{32}$ Dabei wurde eine repräsentative Stichprobe von 19'706 Personen vorerst telefonisch und anschliessend mittels eines Fragebogens schriftlich befragt. Die Rücklaufquote der Fragebögen betrug 82\%.

Diejenigen Befragten, welche innerhalb eines Jahres vor der Befragung Komplementärmedizin in Anspruch genommen hatten, waren durchschnittlich einen halben Tag weniger lang im Spital als Nicht-Anwender (0.9 gegenüber 1.4 Tage) und dies sogar trotz eines schlechteren subjektiven Gesundheitszustandes. Bei Befragten, welche ausschliesslich Komplementärmedizin in Anspruch nahmen, und dies nur bei einem diplomierten Arzt (,reine Anwender"), betrug die Differenz gar 0.9 Tage ( 0.5 gegenüber 1.4 Tage), in diesem Fall jedoch bei vergleichbarem subjektivem Gesundheitszustand. ${ }^{33}$

Allerdings beruhen diese Angaben lediglich auf der subjektiven Erinnerung der Befragten an die Anzahl ihrer Spitalaufenthaltstage in den vergangenen 12 Monaten. Zudem konnte bei den diplomierten Ärzten, bei welchen die Befragten Komplementärmedizin in Anspruch nahmen, nicht unterschieden werden zwischen solchen mit und ohne Fähigkeitsausweis. Auch wurden die Daten zwar nach Geschlecht stratifiziert, aber nicht alterskorrigiert. Dennoch kamen die Autoren zum Schluss, dass komplementärmedizinische Leistungen vor allem bei „reinen Anwendern“ und insbesondere im Hinblick auf die Anzahl Spitalaufenthaltstage substitutiven Charakter haben können. $^{34}$

Diese Einschätzung wird, besonders was die Anzahl der Spitalaufenthaltstage und die damit verbundenen Kosten anbelangt, durch die AMOS-Studie bestätigt, die als prospektive Versorgungsstudie mit Kohortendesign angelegt war. Dabei wurden unter anderem die Gesundheitskosten von 717 Patienten, welche von Januar 1999 bis März 2001 durch 134 anthroposophische Ärzte rekrutiert worden waren, über einen Zeitraum von drei Jahren erfasst und analysiert. Im Vergleich zum Studienvorjahr resultierte im ersten Behandlungsjahr, trotz der neuen anthro-

\footnotetext{
${ }^{30}$ Busato, Eichenberger, Kuenzi, 2006, S. 8f, gestützt auf Busato, Dönges et al.: 2006.

${ }^{31}$ Die von den Patienten im Rahmen der PEK-Praxisstudie II berichteten Nebenwirkungen waren bei Patienten von CAM-Ärzten im Allgemeinen erheblich geringer als bei jenen von COM-Ärzten. Melchart et al., 2005, S. 39. Marian F, Joost K, Saini KD, von Ammon K, Thurneysen A, Busato A: Patient satisfaction and side effects in primary care: an observational study comparing homeopathy and conventional medicine. BMC Complement Altern Med 2008; 8:52.

${ }^{32}$ Crivelli L, Ferrari D, Limoni C: Inanspruchnahme von 5 Therapien der Komplementärmedizin in der Schweiz. Statistische Auswertung auf der Basis der Daten der Schweizerischen Gesundheitsbefragung 1997 und 2002, SUPSI, Scuola Universitaria Professionale della Svizzera italiana, Dipartimento scienze aziendali e sociali, Manno, 30. September 2004.

${ }^{33}$ Crivelli et al., 2004, S. 131f. Was die Zahl der Arztkontakte anbelangt, so lag diese bei den Anwendern von Komplementärmedizin um einen höher als bei den Nichtanwendern (5.4 gegenüber 4.4), allerdings bei subjektiv schlechterem Gesundheitszustand. Bei „,reinen Anwendern“ von Komplementärmedizin, die einen diplomierten Arzt konsultierten, lagen sie jedoch um 1 tiefer (3.4 gegenüber 4.4), bei subjektiv gleichem Gesundheitszustand. Crivelli et al., 2004, S. $120 \mathrm{f}$

${ }^{34}$ Crivelli et al., 2004, S. 121, $131 \mathrm{f}$
} 
posophischen Therapien, kein signifikanter Anstieg der Kosten, während die Kosten im zweiten Studienjahr signifikant um 13\% im Vergleich zum Studienvorjahr sanken. Dieser Rückgang der Kosten liess sich weitgehend auf eine Verringerung der stationären Krankenhauskosten zurückführen. Gleichzeitig zeigten sich signifikante, klinisch relevante und anhaltende Verbesserungen der Krankheitssymptome und -beschwerden. Auch die gesundheitsbezogene Lebensqualität verbesserte sich anhaltend. ${ }^{35}$

Die AMOS-Studie beinhaltete zwar keine Kontrollgruppe; ein Verfahren zur Bias-Suppression zeigte jedoch auch nach Ausschluss von vier möglichen Bias-Faktoren eine signifikante Verbesserung des Krankheitsscores. ${ }^{36}$ Eine weiterführende Kostenanalyse mit 487 erwachsenen AMOS-Patienten ergab, dass die langfristige Kostenverringerung vor allem bei solchen Patienten stattfand, die wegen Depression behandelt wurden - wiederum bei deutlicher Verbesserung der Krankheitsbeschwerden. Auch hier waren es die stationären Behandlungskosten, die sich neben den indirekten Kosten als Folge von Arbeitsunfähigkeit deutlich verringerten. ${ }^{37}$

Demgegenüber zeigte eine vergleichende, prospektive Studie zu den Kosten von homöopathischen und konventionellen Behandlungen über den Zeitraum von einem Jahr keine signifikanten Differenzen bei den Gesamtkosten, aber einen besseren Behandlungserfolg bei den homöopathisch behandelten Patienten. ${ }^{38}$

Sowohl die Auswertung der Schweizerischen Gesundheitsbefragung als auch die AMOS-Studie unterstützen jedenfalls die Hypothese, dass die Kosten von Patienten, die von zertifizierten Ärzten ausschliesslich oder zusätzlich komplementärmedizinisch behandelt werden, im Vergleich zur Behandlung durch COM-Ärzte nicht höher sind.

Umgekehrt ergeben sich wenig Anhaltspunkte für die Hypothese, dass aufgrund unwirksamer Behandlungen die Behandlungskosten andernorts umso höher ausfallen. Die diesbezüglich oft ins Feld geführte Sommer-Studie, ${ }^{39}$ die im Rahmen des NFP-34 Projekts „Komplementärmedizin“ durchgeführt worden war und behauptet hatte, komplementärmedizinische Therapien hätten keine Auswirkungen auf den Gesundheitszustand und führten lediglich zu höheren, weil additiven Kosten, hält einer genaueren Überprüfung nicht stand. ${ }^{40}$ Vielmehr deutete auch sie in ihrem nichtexperimentellen Teil auf substitutive Effekte der Inanspruchnahme komplementärmedizinischer Methoden hin, was aber von den Autoren damals nicht herausgearbeitet und gewürdigt wurde. ${ }^{41}$

\section{Wie objektiv und aussagekräftig sind Selbsteinschätzungen?}

Akzentuiert wird dieses tendenziell positive Zwischenfazit zugunsten der ärztlichen Komplementärmedizin durch die Tatsache, dass der Anteil chronisch Kranker vor allem bei den zertifizierten CAM-Ärzten deutlich höher ist als bei den COM-Ärzten. Als chronisch krank wurde definiert, wer während mehr als drei Monaten von einem Hauptsymptom betroffen war. Zwar könnte argu-

\footnotetext{
${ }^{35}$ Hamre HJ, Witt CM, Glockmann A, Ziegler R, Willich SN, Kiene H: Health costs in anthroposophic therapy users: a two-year prospective cohort study. BMC Health Services Research 2006, 6:65.

${ }^{36}$ Hamre HJ, Glockmann A, Kienle GS, Kiene H: Combined bias suppression in single-arm therapy studies. J Eval Clin Pract. 2008 Oct;14(5):923-9.

${ }^{37}$ Hamre HJ, Witt CM, Glockmann A et al.: Health costs in patients treated for depression, in patients with depressive symptoms treated for another chronic disorder, and in non-depressed patients: a two-year prospective cohort study in anthroposophic outpatient settings. Eur J Health Econ 2010;11(1):77-94.

${ }^{38}$ Witt C, Keil T, Selim D, Roll S, Vance W, Wegscheider K, Willich SN: Outcome and costs of homeopathic and conventional treatment strategies: A comparative cohort study in patients with chronic disorders. Complementary Therapies in Medicine (2005) 13, 79-86.

${ }^{39}$ Sommer JH, Bürgi M, Theiss R: Komplementärmedizin in der Krankenversicherung, Gesundheitsökonomische Analyse der Wirkungen des Einbezuges komplementärmedizinischer Leistungen in die Krankenversicherung, EMH Schweizerischer Ärzteverlag, Basel 1998.

${ }^{40}$ Kienle GS, Keine H: Können aus der Studie „Komplementärmedizin in der Krankenversicherung“ wissenschaftlich tragfähige Schlüsse gezogen werden? Gutachten, Institut für angewandte Erkenntnistheorie und medizinische Methodologie, Freiburg im Breisgau, November 1998.

${ }^{41}$ Vgl. Studer HP: Eine kritische Überprüfung der Nationalfondsstudie „Komplementärmedizin in der Krankenversicherung“ zeigt: Natürliches Heilen spart Kosten und fördert die Gesundheit, SVNH Schweizerischer Verband für Natürliches Heilen, Bern, April 1999, S. $29 \mathrm{ff}$.
} 
mentiert werden, dieses Kriterium sei unscharf, und es sei insbesondere möglich, dass CAMÄrzte im Gegensatz zu COM-Ärzten lediglich leichtere chronische Erkrankungen behandelten. Dem steht jedoch entgegen, dass gemäss Selbsteinschätzung der Patienten der Schweregrad ihrer Erkrankung im Fall von CAM-Patienten deutlich höher liegt als derjenige von COM-Patienten. Dem Argument, eine solche Selbsteinschätzung sei nicht objektiv, kann entgegen gehalten werden, dass gemäss anderen Studien Selbsteinschätzungen durch die Patienten durchaus verlässliche Aussagekraft zukommt. ${ }^{42}$ Auch die Einschätzung durch die Ärzte selbst weist in diese Richtung. Ferner haben sich in anderen, kürzlich durchgeführten Studien zur Grundversorgung im Schweizerischen Gesundheitswesen keine Unterschiede hinsichtlich des subjektiv (durch den Patienten) und des objektiv (durch den Arzt) eingeschätzten Gesundheitszustandes gezeigt. ${ }^{43}$

\section{Sich für die Patienten Zeit nehmen zahlt sich aus}

Der Umstand, dass sich vor allem zertifizierte CAM-Ärzte deutlich mehr Zeit für ihre Patienten nehmen, stellt wohl auch einen wesentlichen Grund für deren höhere Zufriedenheit mit der Behandlung und ihren Begleitumständen dar. ${ }^{44}$

Mit Bezug auf die längere Konsultationsdauer von CAM-Ärzten weisen Busato et al. denn auch darauf hin, dass diese offenbar einen stärker interpersonalen Ansatz der Grundversorgung pflegen. Wenn künftig Strategien des Empowerments der Patienten und einer patientenzentrierten Versorgung verfolgt werden sollen, könnten die Ergebnisse ihrer Studie als Leitlinie verwendet werden, um die Grundversorgung dahingehend zu verbessern, das übergeordnete Ziel einer hocheffektiven interpersonalen Versorgung zu erreichen. ${ }^{45}$

Die Konsultationszeit könne dann - so führen die Autoren weiter aus - als eine Art gemeinsame Währung für eine qualitativ hochstehende Grundversorgung gesehen werden. Die Entscheidung der schweizerischen Gesundheitsbehörden, die ärztliche Komplementärmedizin wieder aus der obligatorischen Krankenpflegeversicherung auszuschliessen, zeige, dass die Wichtigkeit einer patientenzentrierten Grundversorgung vom schweizerischen Gesundheitssystem noch zuwenig anerkannt werde - dies, obwohl die Stärkung der Eigenverantwortung und von patientenzentrierten Ansätzen durch die Schweizerische Akademie der Medizinischen Wissenschaften und andere Institutionen gefördert werde. ${ }^{46}$

Nebst längeren Konsultationszeiten und damit verbundenen intensiveren Arzt-Patienten-Kontakten dürfte aber auch die Tatsache zur höheren Patientenzufriedenheit beigetragen haben, dass komplementärmedizinische Behandlungen im Allgemeinen mit geringeren Nebenwirkungen einhergehen als rein schulmedizinische Diagnosen und Therapien. Auch dahingehend kann der Entscheid der Gesundheitsbehörden, die ärztliche Komplementärmedizin wieder aus der Grundversicherung zu streichen, in Zweifel gezogen werden, stellen doch Nebenwirkungen medizinischer Behandlungen einen gewichtigen Kostenfaktor im Gesundheitswesen dar.

\footnotetext{
${ }^{42}$ Mermod J, Fischer L, Staub L, Busato A: Patient satisfaction of primary care for muscoloskeletal diseases: A comparison between Neural Therapy and conventional medicine, BMC Complementary and Alternative Medicine 2008, 8:33, 24 June 2008, S. 6f, mit Bezug auf: Miilunpalo S, Vuori I, Oja P, Pasanen M, Urponen H: Selfrelated health status as a health measure: the predictive value of self-reported health status on the use of physicians services on mortality in the working-age population. J Clin Epidemiol 1997, 50(5): 517-528 und Hunt SMMKS, Mc Ewen J, Williams J, Papp E: The Nottingham Health Profile: subjective health status and medical consultations. 1981, 15(3 Pt I): 221-229. Vgl. auch Esch BM, Marian F, Busato A, Heusser P: Patient satisfaction with primary care: an observational study comparing anthroposophic and conventional care. BMC Health and Quality of Life Outcomes 2008, 6:74, 30. Sept. 2008, S. 1-15.

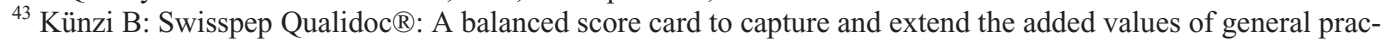
tice/family medicine. Houten, NL: Bohn Stafleu van Lohum; 2004.

${ }^{44} \mathrm{Vgl}$. Wilson A, Childs S: The relationship between consultation length, process and outcomes in general practice: a systematic review. British Journal of General Practice, 52 (485), S. 1012-1020, sowie Martin C, Banwell $\mathrm{CL}$, Broom DH, Nisa M: Consultation length and chronic illness care in general practice: a qualitative study. Medical Journal of Autralia, 171 (2), S. 77-81.

${ }^{45}$ Busato, Eichenberger, Kuenzi, 2006, S. 10.

${ }^{46}$ Busato, Eichenberger, Kuenzi, 2006, S. 10f, unter anderem mit Bezug auf Schweizerische Akademie der Medizinischen Wissenschaften (SAMW): Ziele und Aufgaben der Medizin zu Beginn des 21. Jahrhunderts, 2004.
} 


\section{Schlussfolgerungen}

Mit der Kombination von Schul- und Komplementärmedizin praktizieren zertifizierte CAM-Ärzte offenbar eine Form der Integrativen Medizin, bei der sich die längeren Konsultationszeiten auch pro Patient nicht in höheren Kosten niederschlagen. Deswegen und weil sich des Weiteren bezüglich verschiedener nutzenbezogener Kriterien Vorteile für die ärztliche Komplementärmedizin zeigten, erscheint deren Wirtschaftlichkeit aufgrund unserer Daten gegeben.

Dies ist insbesondere dann der Fall, wenn der Miteinbezug der Komplementärmedizin in die obligatorische Krankenpflegeversicherung an die Voraussetzung eines FMH-anerkannten Fähigkeitsausweises und einer entsprechenden methodenspezifischen Zusatzausbildung geknüpft wird. Zertifizierte CAM-Ärzte schnitten jedenfalls bei gleichen Kosten in Bezug auf nutzenbezogenen Kriterien gegenüber den nicht-zertifizierten CAM-Ärzten besser ab.

Allerdings sollten die vorliegenden Erkenntnisse durch zusätzliche Forschung vertieft werden, vor allem mit dem Ziel, einerseits die patientenbezogenen Kosten umfassender zu erheben und andererseits die Zusammenhänge mit der Art und dem Schweregrad der Erkrankung noch gezielter zu erfassen und auszuwerten. Bereits vor dem Hintergrund unserer Ergebnisse erscheint es jedoch aus dem Blickwinkel der Wirtschaftlichkeit durchaus gerechtfertigt, der ärztlichen Komplementärmedizin in der obligatorischen Krankenpflegeversicherung wieder ihren Platz einzuräumen.

Im Zusammenhang damit ist ferner darauf hinzuweisen, dass der Miteinbezug der ärztlichen Komplementärmedizin in die obligatorische Krankenpflegeversicherung auch aus Sicht der Patienten und Versicherten wünschenswert erscheint. Zum einen erhalten so Patienten, die über keine komplementärmedizinische Zusatzversicherung verfügen und eine Therapie nicht selber bezahlen könnten, ebenfalls Zugang zu einer komplementärmedizinischen Grundversorgung. Und zum anderen wird das Versicherungskollektiv dadurch kaum zusätzlich finanziell belastet.

\section{Danksagung}

Die beiden Autoren bedanken sich beim Bundesamt für Gesundheit für die Finanzierung des PEK-Forschungsprojekts und bei santésuisse für die Zurverfügungstellung von Kostendaten für diese Studie. Dank geht auch an Andreas Dönges, Antoinette Kearns, Barbara Schmitter, Florica Marian, Kathrin Dopke and Sylvia Herren für ihre Mitarbeit an diversen Teilstudien des Projekts.

\section{Literaturverzeichnis}

Busato A, Dönges A, Herren S, Widmer M, Marian F: Health status and health care utilisation of patients in complementary and conventional primary care in Switzerland - an observational study. Family practice, VOL: 23(1), 2006, 116-124.

Busato A, Eichenberger R, Kuenzi B: Extent and structure of health insurance expenditures for complementary and alternative medicine in Swiss primary care. BMC Health Services Research 2006, 6:132, 1-19.

Busato A, Künzi B: Differences in the quality of interpersonal care in complementary and conventional medicine, im Januar 2010 zur Publikation eingereicht, BMC Complementary and Alternative Medicine.

Crivelli L, Ferrari D, Limoni C: Inanspruchnahme von 5 Therapien der Komplementärmedizin in der Schweiz. Statistische Auswertung auf der Basis der Daten der Schweizerischen Gesundheitsbefragung 1997 und 2002, SUPSI, Scuola Universitaria Professionale della Svizzera italiana, Dipartimento scienze aziendali e sociali, Manno, 30. September 2004.

Eidgenössisches Departement des Innern: Erläuterungen Entscheid komplementärmedizinische Methoden, Bern, 3. Juni 2005 
Eidgenössisches Volkswirtschaftsdepartement, Staatssekretariat für Wirtschaft SECO: Leitfaden Inspektions-Prüfmittel Gesundheitsrisiken Bewegungsapparat, überarbeitete Fassung, Bern, August 2008.

Esch BM, Marian F, Busato A, Heusser P: Patient satisfaction with primary care: an observational study comparing anthroposophic and conventional care. BMC Health and Quality of Life Outcomes 2008, 6:74, 30. Sept. 2008, 1-15.

Foppa D, Mäder Ph: Alternativmedizin: Couchepin räumt Fehler ein, in: Tages-Anzeiger, 30. Januar 2009.

Geschäftsprüfungskommissionen und Geschäftsprüfungsdelegation der eidgenössischen Räte: Jahresbericht 2008, Bern, 23. Januar 2009.

Hamre HJ, Witt CM, Glockmann A, Ziegler R, Willich SN, Kiene H; Health costs in anthroposophic therapy users: a two-year prospective cohort study. BMC Health Services Research 2006, 6:65, 351-360.

Hamre HJ, Glockmann A, Kienle GS, Kiene H: Combined bias suppression in single-arm therapy studies. J Eval Clin Pract. 2008 Oct;14(5):923-9.

Hamre HJ, Witt CM, Glockmann A et al. Health costs in patients treated for depression, in patients with depressive symptoms treated for another chronic disorder, and in non-depressed patients: a two-year prospective cohort study in anthroposophic outpatient settings. Eur J Health Econ 2010;11(1):77-94.

Hunt SMMKS, Mc Ewen J, Williams J, Papp E: The Nottingham Health Profile: subjective health status and medical consultations. 1981, 15(3 Pt I): 221-229.

Kienle GS, Keine H: Können aus der Studie „Komplementärmedizin in der Krankenversicherung“ wissenschaftlich tragfähige Schlüsse gezogen werden? Gutachten, Institut für angewandte Erkenntnistheorie und medizinische Methodologie, Freiburg im Breisgau, November 1998.

Künzi B: Swisspep Qualidoc®: A balanced score card to capture and extend the added values of general practice/family medicine. Houten, NL: Bohn Stafleu van Lohum; 2004.

Marian F, Joost K, Saini KD, von Ammon K, Thurneysen A, Busato A: Patient satisfaction and side effects in primary care: an observational study comparing homeopathy and conventional medicine. BMC Complement Altern Med 2008; 8:52, 1-10.

Martin C, Banwell CL, Broom DH, Nisa M: Consultation length and chronic illness care in general practice: a qualitative study. Medical Journal of Autralia, 171 (2), 77-81.

Matter-Walstra K, Schoeni-Affolter F, Widmer M, Busato A: Patient-based evaluations of primary care for cardiovascular diseases: a comparison between conventional and complementary medicine. Journal of Evaluation in Clinical Practice 14 (2008) 75-82.

Melchart D, Mitscherlich F, Amiet M, Eichenberger R, Koch P: Programm Evaluation Komplementärmedizin, Schlussbericht, Bern, 14. April 2005.

Mermod J, Fischer L, Staub L, Busato A: Patient satisfaction of primary care for muscoloskeletal diseases: A comparison between Neural Therapy and conventional medicine, BMC Complementary and Alternative Medicine 2008, 8:33, 24 June 2008, 1-10.

Michlig M, Ausfeld-Hafter B, Busato A: Patient satisfaction with primary care: A comparison between conventional care and traditional Chinese medicine, Complement Ther Med 2008, 16(6):350-358.

Miilunpalo S, Vuori I, Oja P, Pasanen M, Urponen H: Self-related health status as a health measure: the predictive value of self-reported health status on the use of physicians services on mortality in the workingage population. J Clin Epidemiol 1997, 50(5): 517-528.

Schweizerische Akademie der Medizinischen Wissenschaften (SAMW): Ziele und Aufgaben der Medizin zu Beginn des 21. Jahrhunderts, 2004. 
Sommer JH, Bürgi M, Theiss R: Komplementärmedizin in der Krankenversicherung, Gesundheitsökonomische Analyse der Wirkungen des Einbezuges komplementärmedizinischer Leistungen in die Krankenversicherung, EMH Schweizerischer Ärzteverlag, Basel 1998.

Studer HP: Eine kritische Überprüfung der Nationalfondsstudie „Komplementärmedizin in der Krankenversicherung“ zeigt: Natürliches Heilen spart Kosten und fördert die Gesundheit, SVNH Schweizerischer Verband für Natürliches Heilen, Bern, April 1999.

Wallach H, Linde K, Eichenberger R, Stalder H, Kristensen FB, Kleijnen J: Konsens-Stellungnahme des PEK-Review Boards zum Prozess und den Produkten von PEK, 27. September 2005.

Wapf V, Busato A: Patients' motives for choosing a physician: comparison between conventional and complementary medicine in Swiss primary care, BMC Complementary and Alternative Medicine 2007, $7: 41,1-10$.

Wilson A, Childs S: The relationship between consultation length, process and outcomes in general practice: a systematic review. British Journal of General Practice, 52 (485), 1012-1020.

Witt C, Keil T, Selim D, Roll S, Vance W, Wegscheider K, Willich SN: Outcome and costs of homeopathic and conventional treatment strategies: A comparative cohort study in patients with chronic disorders. Complementary Therapies in Medicine (2005) 13, 79-86. 